\title{
Gabapentinoid treatment promotes corticospinal plasticity and regeneration following murine spinal cord injury
}

\author{
Wenjing Sun, ${ }^{1}$ Molly J.E. Larson, ${ }^{1}$ Conrad M. Kiyoshi, ${ }^{1}$ Alexander J. Annett, ${ }^{1}$ William A. Stalker, ${ }^{1}$ Juan Peng, ${ }^{2}$ and Andrea Tedeschi ${ }^{1,3}$ \\ 'Department of Neuroscience, Wexner Medical Center, ${ }^{2}$ Center for Biostatistics and Bioinformatics, and ${ }^{3}$ Chronic Brain Injury Program, The Ohio State University, Columbus, Ohio, USA
}

\begin{abstract}
Axon regeneration failure causes neurological deficits and long-term disability after spinal cord injury (SCI). Here, we found that the $\alpha 2 \delta 2$ subunit of voltage-gated calcium channels negatively regulates axon growth and regeneration of corticospinal neurons, the cells that originate the corticospinal tract. Increased $\alpha 2 \delta 2$ expression in corticospinal neurons contributed to loss of corticospinal regrowth ability during postnatal development and after SCI. In contrast, $\alpha \mathbf{2} \delta 2$ pharmacological blockade through gabapentin administration promoted corticospinal structural plasticity and regeneration in adulthood. Using an optogenetic strategy combined with in vivo electrophysiological recording, we demonstrated that regenerating corticospinal axons functionally integrate into spinal circuits. Mice administered gabapentin recovered upper extremity function after cervical SCI. Importantly, such recovery relies on reorganization of the corticospinal pathway, as chemogenetic silencing of injured corticospinal neurons transiently abrogated recovery. Thus, targeting $\alpha \mathbf{2 \delta 2}$ with a clinically relevant treatment strategy aids repair of motor circuits after SCl.
\end{abstract}

\section{Introduction}

Injuries to the adult spinal cord disrupt communication between the brain and the spinal cord, causing neurological deficits and longterm disability. In about half of individuals with spinal cord injury (SCI), the injury occurs at cervical levels (1), compromising function of upper and lower extremities and severely impairing daily activities that are essential for independent living. Thus far, no therapeutic strategy that restores function is available for individuals with SCI.

Recovery of spinal cord function in adults might be attained by promoting axon sprouting, regeneration, and de novo formation of neural circuits (2-4). Among the descending pathways important for SCI repair, the corticospinal tract exerts the sensory and motor control that is necessary for accurate limb placement and voluntary movements (5). In the adult central nervous system (CNS), however, injured neurons have limited capacity to sprout and even less to regenerate, often failing to reestablish functionally meaningful connections (6). Several strategies have recently challenged this notion by reprogramming the deficient intrinsic growth state of adult neurons and eliminating extracellular growth inhibitors, triggering regrowth of axonal tracts that project to the spinal cord (7-14). A major challenge is that implementation of these strategies might not be immediately available for clinical interventions.

In exploring the mechanisms that control intrinsic axon growth ability, we previously discovered that the $\alpha 2 \delta 2$ subunit of voltage-gated calcium channels suppresses axon growth and regeneration in adult mouse sensory neurons, and that $\alpha 2 \delta 2$ pharmaco-

Conflict of interest: The authors have declared that no conflict of interest exists. Copyright: (5) 2020, American Society for Clinical Investigation.

Submitted: May 20, 2019; Accepted: October 2, 2019; Published: December 3, 2019.

Reference information: J Clin Invest. 2020;130(1):345-358.

https://doi.org/10.1172/JCl130391. logical blockade through administration of gabapentinoids (e.g., pregabalin or gabapentin), drugs used clinically to treat neurological disorders $(15,16)$, promoted regeneration of ascending sensory axons after SCI (17). Thus far, our mechanistic understanding of whether CNS motor pathways other than sensory ascending fibers respond to the same treatment strategy remains limited. In addition, it is unclear whether gabapentinoid-dependent reorganization of motor circuits aids functional recovery after SCI.

Here, we show that $\alpha 2 \delta 2$ is expressed in corticospinal neurons, the cells that originate the corticospinal tract. Whereas $\alpha 2 \delta 2$ negatively regulates corticospinal axon regrowth ability during postnatal development and after SCI, gabapentin (GBP) administration promoted sprouting and regeneration of corticospinal axons in adulthood. These regenerating corticospinal axons functionally integrate into spinal circuits, effectively promoting recovery of upper extremity function after cervical SCI in mice administered GBP. Thus, our study highlights the strong potential for repurposing gabapentinoids as a novel treatment for SCI repair.

\section{Results}

The $\alpha 2 \delta 2$ subunit is expressed in corticospinal neurons and subjected to developmental- and postinjury-dependent upregulation. We first determined whether $\alpha 2 \delta 2$ was expressed in corticospinal neurons. We injected the retrograde tracer Fluoro-Gold into the cervical spinal cord of adult transgenic mice with sparse green fluorescent protein (GFP) expression in a variety of neuronal subsets, including corticospinal neurons (ref. 18 and Figure 1A). Three days later, we stained sagittal sections of the brains that contained retrogradely labeled corticospinal neurons with a polyclonal antibody that recognizes murine $\alpha 2 \delta 2$. Corticospinal neurons that projected to the cervical spinal cord expressed $\alpha 2 \delta 2$ (Figure 1B). Previous work used high-throughput analysis to profile gene expression in 
A

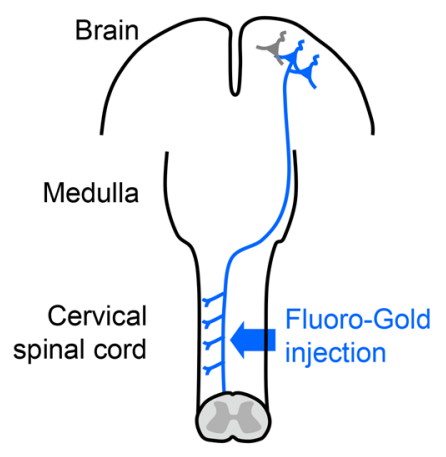

C

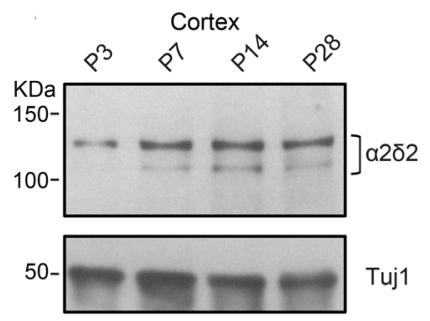

G

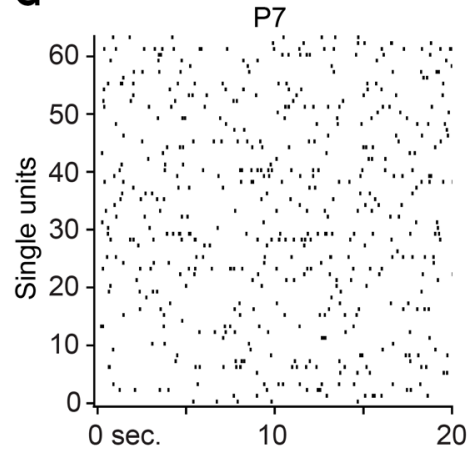

B

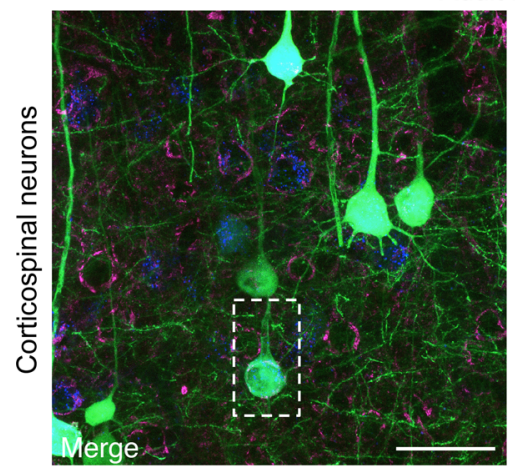

E

D

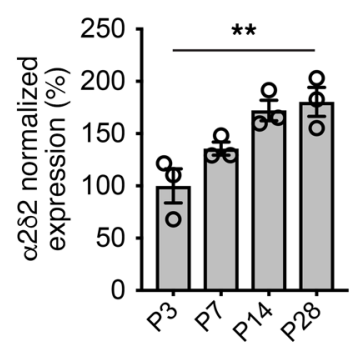

Adult mouse brain (GFP-M mice)

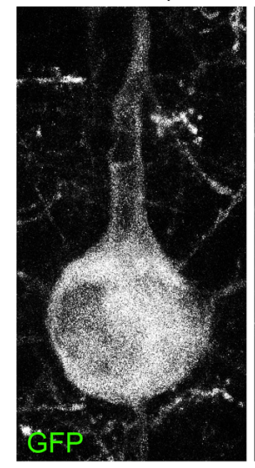

Corticospinal neurons
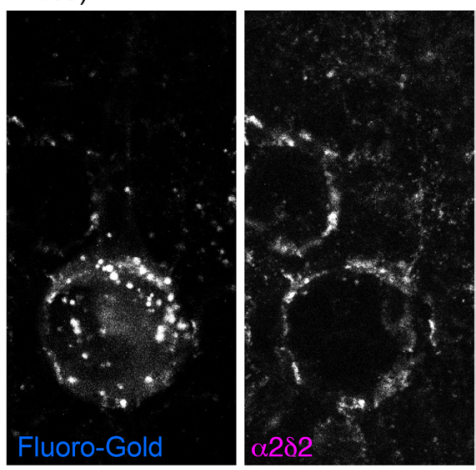

$\mathbf{F}$
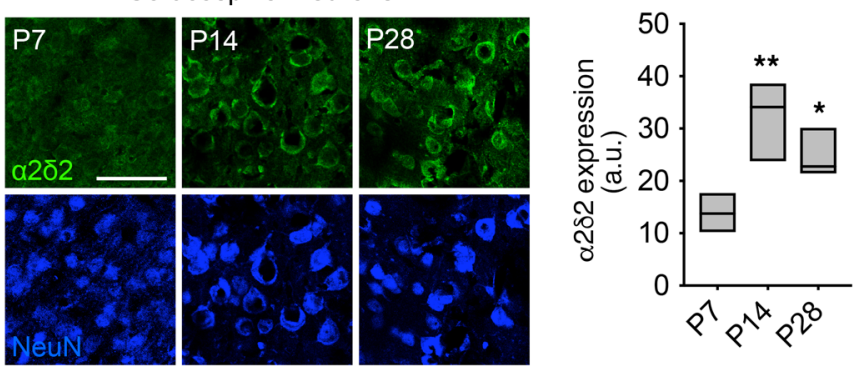
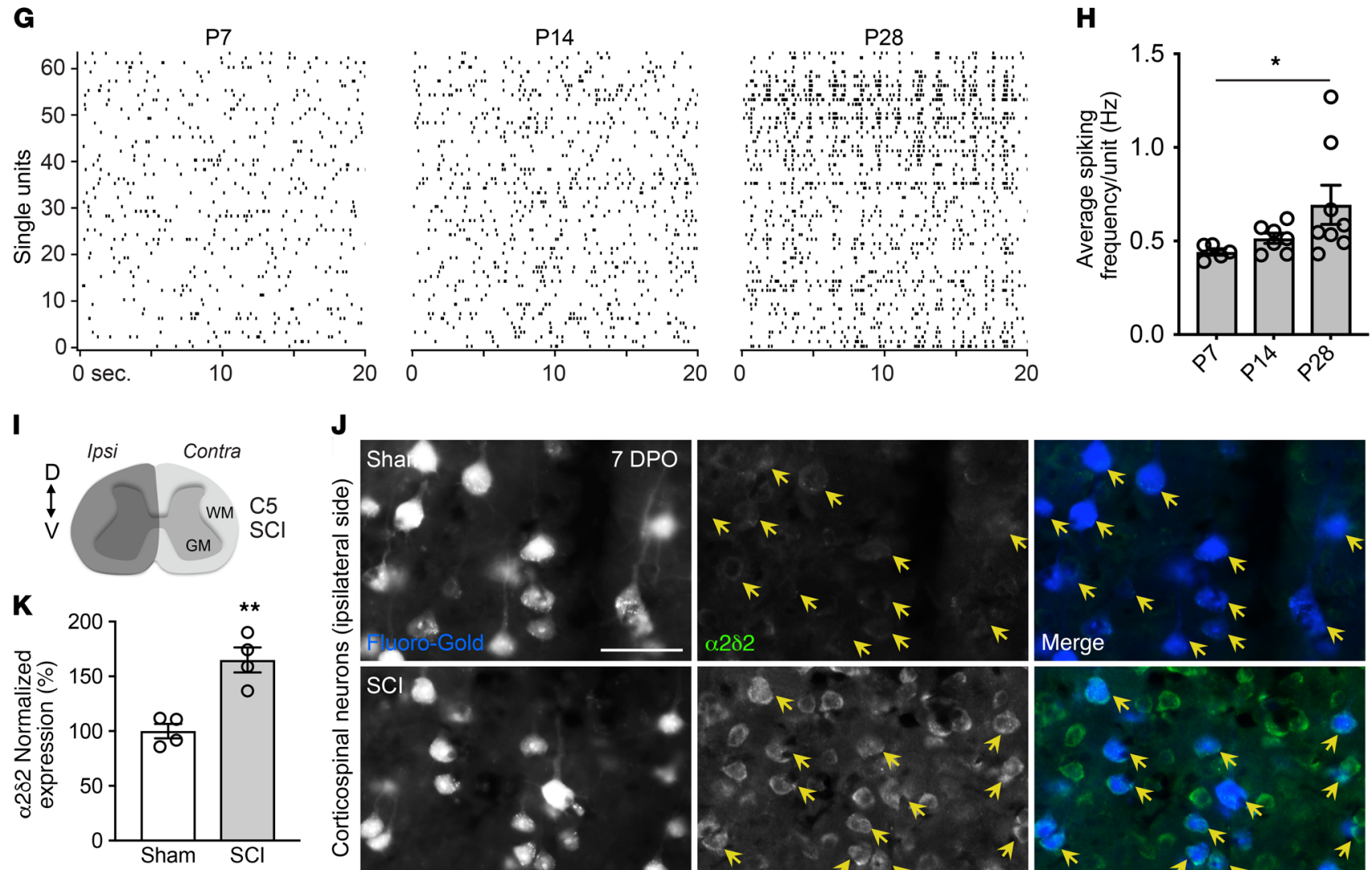

\section{J}
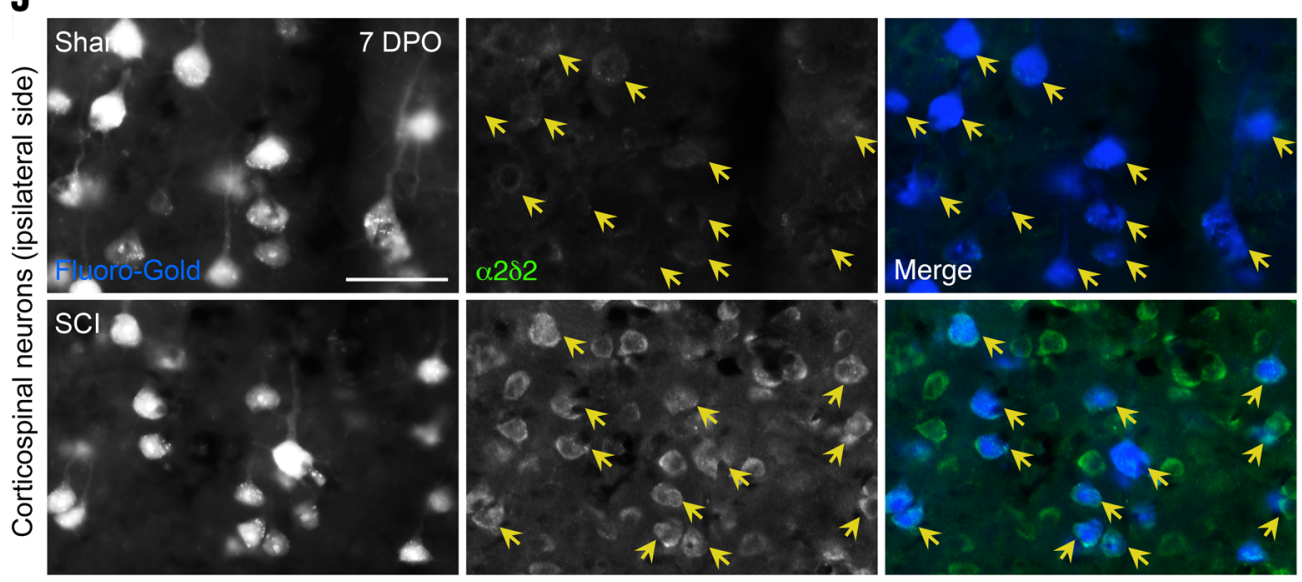
Figure 1. $\alpha 2 \delta 2$ is expressed in corticospinal neurons and subjected to developmental and injury-dependent upregulation. (A) Schematic of retrograde labeling of corticospinal neurons. (B) Representative fluorescence images of corticospinal neurons identified by retrograde labeling after Fluoro-Gold injections into the cervical spinal cords of adult GFP-M mice. Sagittal sections of the mouse brain were immunostained with $\alpha 2 \delta 2$ antibody ( $n=4$ independent replicates). Scale bar: $50 \mu \mathrm{m}$. (C) Immunoblot shows $\alpha 2 \delta 2$ expression in the mouse sensory-motor cortex during postnatal development. Under reducing conditions, the $\alpha 2 \delta 2$ antibody recognizes 2 bands at approximately 130 and $105 \mathrm{kDa}$. Tuj is used as loading control. (D) Quantification of C. Data normalized using loading control (linear trend test ${ }^{* *} P<0.01, n=3$ biological replicates). (E) Representative fluorescence images of corticospinal neurons from mouse brains at different ages. Scale bar: $50 \mu \mathrm{m}$. (F) Quantification of E. Box plot (minimum to maximum) and line at median (1-way ANOVA followed by Dunnett post test ${ }^{*} P<0.05 ;{ }^{* *} P<0.01 ; P 7 n=5, P 14 n=3$, and P28 $n=3$ mice, 60-95 neurons per condition). (G) Raster plots show spontaneous firing within layer $V$ of the sensory-motor cortex at different stages of brain development. (H) Quantification of G. Mean and SEM (linear trend test ${ }^{*} P<0.05$; P7 $n=5$, P14 $n=7$, and P28 $n=8$ mice). (I) Schematic representation of C5 SCl experimental model. (J) Representative fluorescence images of retrogradely labeled corticospinal neurons (yellow arrows) 7 days after $C 5$ SCI. DPO, days after operation. Sagittal sections of the mouse brain (right hemisphere) were immunostained with $\alpha 2 \delta 2$ antibody. Scale bar: $50 \mu \mathrm{m}$. (K) Quantification of J. Mean and SEM (unpaired 2-tailed Student's $t$ test ${ }^{*} P<0.01$; sham $n=4$ and $\mathrm{SCI} n=4$ mice, 229-302 neurons per condition).

FACS-purified callosal, corticospinal, and corticothalamic neurons during development (19). Starting from this publicly available RNA-sequencing data, we discovered that Cacna2d2, the gene encoding $\alpha 2 \delta 2$, was developmentally upregulated in corticospinal neurons (http://decon.fas.harvard.edu/pyramidal/gene/ Cacna2d2). Our analysis showed that expression of Cacna2d2 and $\alpha 2 \delta 2$ increased in the sensory-motor cortex and corticospinal neurons during postnatal development in mice (Figure 1, C-F, and Supplemental Figure 1A; supplemental material available online with this article; https://doi.org/10.1172/JCI130391DS1).

In different systems and pathological conditions, $\alpha 2 \delta$ subunits positively regulate synaptic properties and neurotransmission (17, 20-22). To determine whether increased $\alpha 2 \delta 2$ expression parallels with changes in electrical properties of developing corticospinal neurons, we recorded spontaneous firing within layer $\mathrm{V}$ in vivo using multichannel electrode arrays at P7, P14, and P28. We found increased neuronal spiking activity at P14 and P28 when compared with P7 (Figure 1, G and H, and Supplemental Figure 1, B and C). In adult mice, increased spontaneous firing of corticospinal neurons is associated with maladaptive plasticity after SCI (23). We discovered that $\alpha 2 \delta 2$ expression increased in adult corticospinal neurons 7 days after a cervical 5 (C5) SCI that completely severed corticospinal axons (Figure 1, I-K, and Supplemental Figure 1D). In contrast, a reduction in $\alpha 2 \delta 2$ expression was found in the contralateral brain hemisphere (Supplemental Figure 1, E and F). Thus, increased $\alpha 2 \delta 2$ expression parallels with modifications of intrinsic properties of corticospinal neurons and augmented network activity during nervous system development and after SCI.

Corticospinal sprouting and axon growth are negatively regulated by $\alpha 2 \delta 2$. Given that sprouting and regeneration of the corticospinal tract is very limited in adults and that immature neurons possess extraordinary axon growth and regeneration ability (11, 24), we searched for a possible causal relationship between the intrinsic growth state of corticospinal neurons and $\alpha 2 \delta 2$ expression. We performed a unilateral pyramidotomy (PTX) to sever the left corticospinal tract in the medullary region rostral to the pyramidal decussation at P10 (Figure 2A). At this time of brain development, $\alpha 2 \delta 2$ expression in the sensory-motor cortex was lower compared with that at P28. At 4 weeks of age, we injected an anterograde tracer, biotinylated dextran amine (BDA), into the right sensory-motor cortex to label the uninjured corticospinal tract on the contralateral side. In sham controls, the majority of labeled corticospinal axons projected ipsilaterally (Figure 2B). In contrast, numerous corticospinal axons sprouted into the contralateral denervated side of the cervical spinal cord after unilateral PTX (Figure 2, B-E). Sprouting corticospinal neurons had reduced $\alpha 2 \delta 2$ expression when compared with the sham controls (Figure 2, F and G), suggesting an inverse correlation between structural corticospinal plasticity and $\alpha 2 \delta 2$ expression. Of note, sprouting axons established new synaptic contacts, as shown by the presence of the BDA-labeled axons expressing the presynaptic marker vesicular glutamate transporter 1 (VGLUT1) in close opposition to the postsynaptic marker Homer1 (Figure 2H).

To determine whether $\alpha 2 \delta 2$ negatively regulates axon growth in immature cortical neurons, we forced expression of Cacna $2 \mathrm{~d} 2$ by electroporating E17.5 mouse cortical neurons in vitro using GFP with either control plasmids or Cacna2d2-expressing plasmids (Figure 2I). Control electroporated neurons extended long neurites 2 days after plating, whereas Cacna2d2-overexpressing neurons extended shorter neurites (Figure 2, J and K). When cultured in the presence of GBP $(250 \mu \mathrm{M})$ to block $\alpha 2 \delta 2$, Cacna $2 d 2$ overexpressing neurons grew neurites to a similar extent as the control condition (Supplemental Figure 2, A and B). Importantly, GBP failed to rescue axon growth defects in neurons overexpressing a mutant $\alpha 2 \delta 2$ that does not bind GBP (ref. 25 and Supplemental Figure 2, A and B), indicating that GBP action on axon growth is mediated through $\alpha 2 \delta 2$ binding.

Given that $\alpha 2 \delta 2$ was expressed in the embryonic sensorymotor cortex and in dissociated and cultured E17.5 cortical neurons (Supplemental Figure 2, C-G), we asked whether GBP increases axon growth of embryonic cortical neurons under normal physiological conditions. When cultured for 2 days in the presence of GBP $(250 \mu \mathrm{M})$ to block $\alpha 2 \delta 2$, embryonic cortical neurons extended longer neurites compared with the control condition (Figure 2, L and $\mathrm{M})$. We therefore asked whether $\alpha 2 \delta 2$ pharmacological blockade in vivo is sufficient to promote corticospinal sprouting in adulthood. Adult mice were subjected to unilateral PTX severing of the left corticospinal tract in the medullary region rostral to the pyramidal decussation. We chronically administered vehicle (0.9\% saline) or GBP (46 mg/kg body weight) starting 1 hour after injury until the end of the study (Figure 3A). Two weeks after injury, the anterograde tracer BDA was injected into the right sensory-motor cortex as described above. Limited or no sprouting was found in control mice (Figure 3B). In contrast, we found extensive collateral sprouting of adult corticospinal axons into the denervated side of the spinal cord in mice receiving GBP (Figure 3, B-E).

GBP had no effect on cortical survival after PTX (Supplemental Figure 3, A and B). Interestingly, $\alpha 2 \delta 2$ expression was lower in the contralateral side of the brain in both experimental conditions, further underscoring an inverse correlation between structural 
A
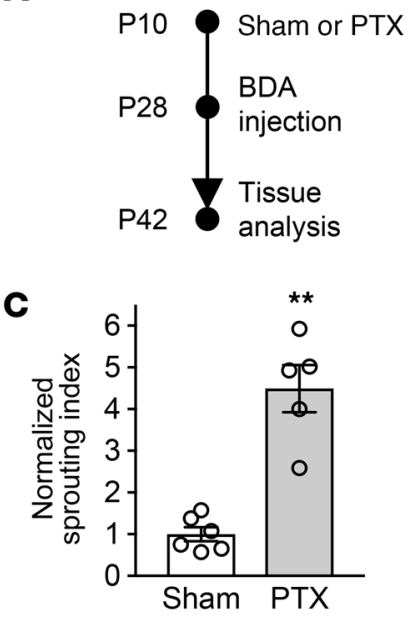

D

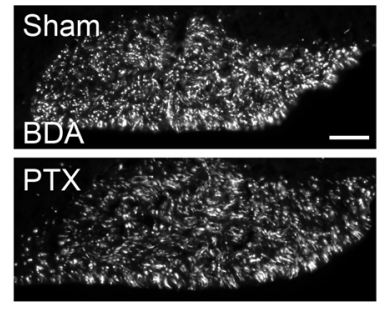

B
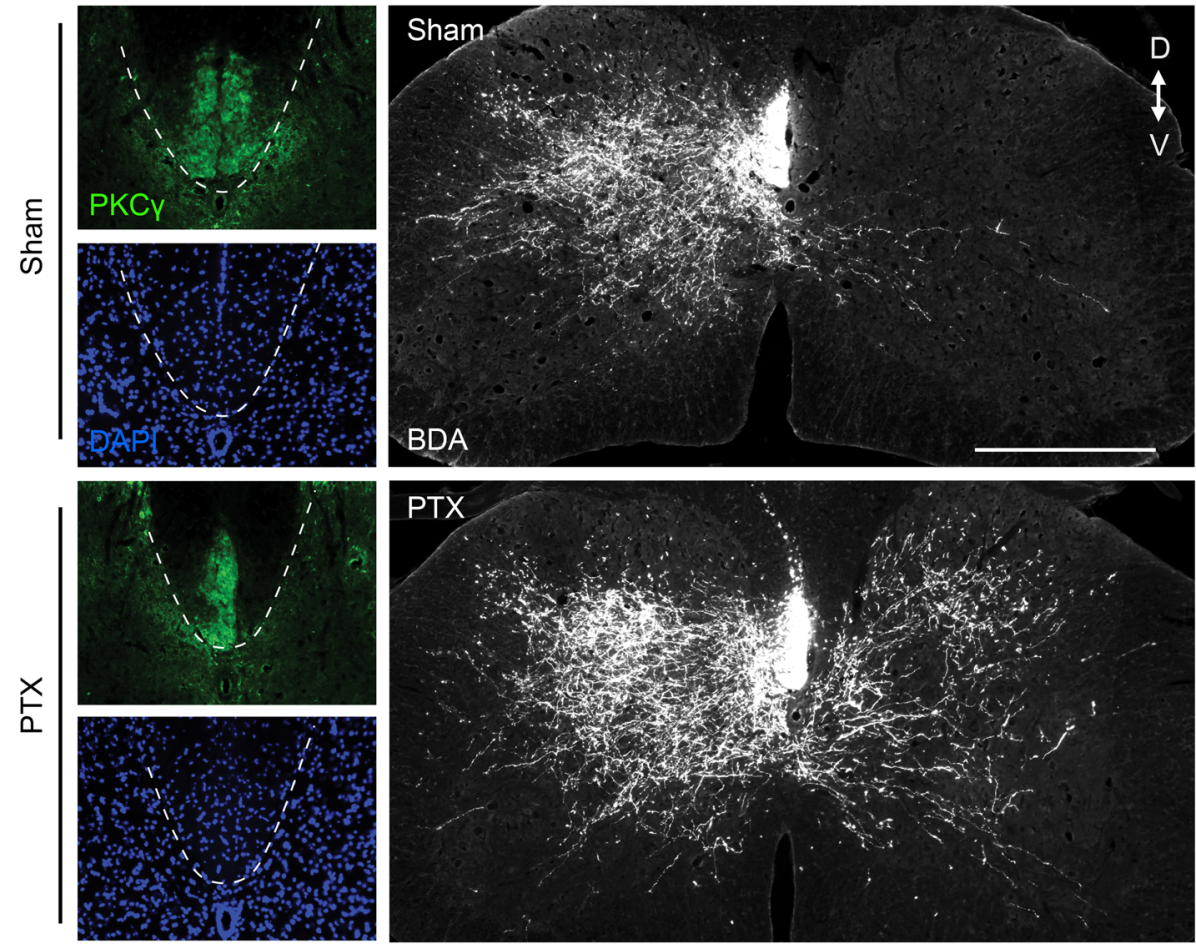

G
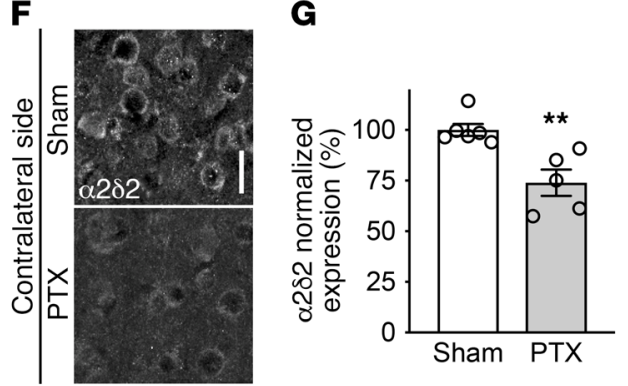

H

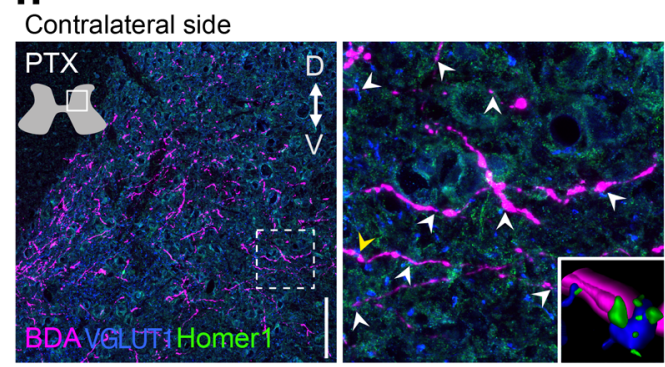

$\mathbf{K}$

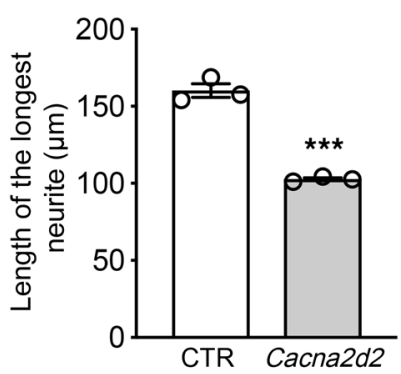

$\mathbf{L}$

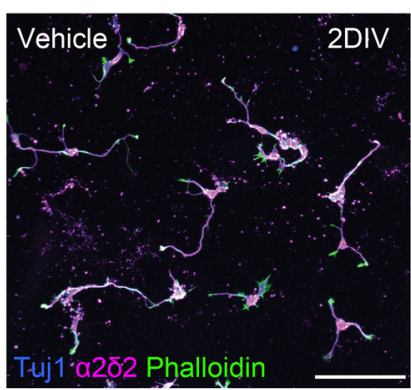

J

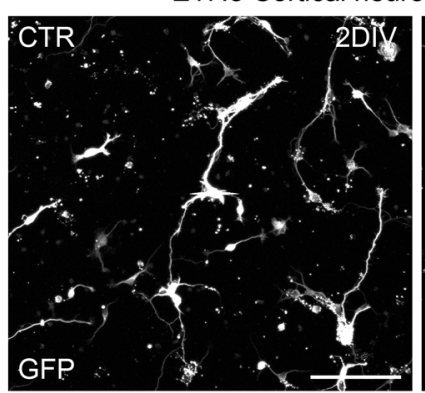

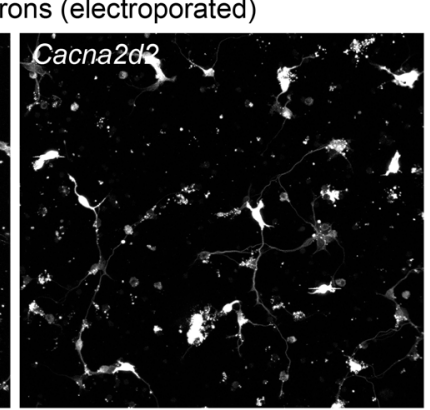

M

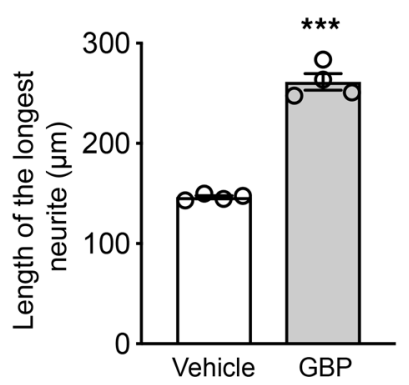


Figure 2. $\alpha \mathbf{2} \delta \mathbf{2}$ inhibits axon growth of cortical neurons. (A) Experimental scheme of B. (B) Representative fluorescence images of C7 spinal cord sections from mice with sham operation or unilateral PTX performed at P10. PKC $\gamma$ staining is shown to confirm lesion completeness. Scale bar: $500 \mu \mathrm{m}$. D, dorsal; V, ventral. (C) Quantification of B. Mean and SEM (unpaired 2-tailed Student's $t$ test ${ }^{*} P<0.01$; sham $n=6$ and PTX $n=5$ mice). (D) Coronal sections of the medullary pyramid showing BDA-labeled corticospinal axons. Scale bar: $50 \mu \mathrm{m}$. (E) Quantification of $\mathbf{D}$ (unpaired 2-tailed Student's $t$ test NS, not significant; sham $n=6$ and PTX $n=5$ mice). (F) Representative fluorescence images of corticospinal neurons 32 days after sham or PTX at P10. Sagittal sections of the mouse brain (right hemisphere) were immunostained with $\alpha 2 \delta 2$ antibody. Scale bar: $25 \mu \mathrm{m}$. (C) Quantification of $\mathbf{F}$ (unpaired 2-tailed Student's $t$ test ${ }^{*} P<0.01$; sham $n=6$ and PTX $n=5$ mice; 278-369 neurons per condition). (H) Representative fluorescence images of C7 spinal cord sections from mice with unilateral PTX performed at P10. The arrows indicate excitatory synaptic puncta along sprouting corticospinal axons. Inset, 3D reconstruction of the region in the main panel indicated by the yellow arrow. Scale bar: $100 \mu \mathrm{m}$. (I) Immunoblot showing $\alpha 2 \delta 2$ expression in E17.5 cortical neurons cultured for 48 hours after electroporation with GFP plus either control (CTR) or Cacna2d2-expressing plasmids. Tuj 1 is shown as a loading control $(n=3$ independent replicates per condition). (J) Representative fluorescence images of E17.5 cortical neurons cultured for 48 hours after electroporation with GFP plus either CTR or Cacna2d2-expressing plasmids. DIV, day in vitro. Scale bar: $100 \mu \mathrm{m}$. (K) Quantification of J. Mean and SEM (unpaired 2-tailed Student's $t$ test ${ }^{* * *} P<0.001$; triplicate experiments; 134-144 neurons per condition). (L) Representative fluorescence images of E17.5 cortical neurons cultured for 48 hours in the presence of either vehicle (0.9\% saline) or $250 \mu$ M GBP. Scale bar: $100 \mu \mathrm{m}$. (M) Quantification of $\mathbf{L}$. Mean and SEM (unpaired 2-tailed Student's $t$ test ${ }^{* * *} P<0.001 ; 4$ independent experiments; 163-165 neurons per condition).

plasticity of corticospinal neurons and $\alpha 2 \delta 2$ expression (Figure 3 F). Of note, trans-midline sprouting axons formed new synaptic structures within appropriate regions of the spinal gray matter in mice receiving GBP (Figure 3G). Taken together, these data support the hypothesis that $\alpha 2 \delta 2$ negatively regulates axon growth in cortical neurons and that $\alpha 2 \delta 2$ pharmacological blockade promotes structural corticospinal plasticity after injury in adulthood.

Pharmacological blockade of $\alpha 2 \delta 2$ promotes structural and functional reorganization of the corticospinal tract after SCI. Multiple axon repair strategies, such as sprouting and regeneration, are required to maximize chances for neurological recovery after SCI (26). We thus asked whether $\alpha 2 \delta 2$ pharmacological blockade would be sufficient to promote corticospinal regeneration and formation of new functional circuits after SCI. To this end, we subjected adult mice to a left C5 lateral hemisection of the spinal cord that completely severed corticospinal axons on 1 side (Supplemental Figure 4, A-C). GBP (46 mg/kg body weight) or an equal amount of vehicle was administered starting 1 hour after injury until the end of the study. To trace and optically stimulate corticospinal axons, we injected adeno-associated viral (AAV) particles expressing a fusion of channelrhodopsin 2 and enhanced yellow fluorescent protein, ChR2(H134R)-eYFP, into the right forelimb sensory-motor cortex 3 months after SCI (Supplemental Figure 5A).

One month later, we recorded the evoked local field potentials (LFPs) upon laser stimulation of ChR2-labeled corticospinal axons in the cervical spinal cord of anesthetized SCI mice (Supplemental Figure 5B). We positioned the multichannel recording electrodes in 3 locations: 2 ipsilaterally $(500 \mu \mathrm{m}$ caudal and rostral to the site of injury) and 1 contralaterally $(500 \mu \mathrm{m}$ rostral to the site of injury). Recording LFPs ipsilaterally/rostrally to the lesion site in both experimental groups served as a positive control for the stimulation parameters. In both groups, laser stimulation evoked little or no response caudal to the lesion (Supplemental Figure 5C). On the contralateral side, instead, larger LFPs were recorded in GBP-treated mice compared with controls (Figure 4, A and B).

At the end of the in vivo recording, mice were transcardially perfused and the spinal cords were dissected. We cleared and imaged the unsectioned spinal cords to visualize labeled corticospinal axons. We observed limited corticospinal regeneration across the lesion site (Supplemental Figure 5, D-F), further supporting the in vivo recording data. Yet, extensive corticospinal regeneration proceeded toward the contralateral side in GBP-treated mice but not in controls (Figure 4, C and D, and Supplemental Figure 5G). Three-dimensional reconstruction of corticospinal trajectories in mice receiving GBP allowed us to visualize numerous regenerating axons with synaptic boutons in the same location where recording electrodes were inserted (Figure 4C), providing further evidence for increased functional connectivity within the injured spinal cord in mice treated with GBP. In a separate cohort, we electrically stimulated the right sensory-motor cortex 4 months after SCI to trigger postsynaptic activity in the injured spinal cord (Figure $4 \mathrm{E}$ and Supplemental Figure 6, A and B). By mapping immediate-early gene activity as a surrogate of functional connectivity, we found increased bilateral c-Fos immunoreactivity in neurons within the $\mathrm{C} 2-\mathrm{C} 4$ spinal segments (e.g., rostral to the injury site) in mice administered GBP (Figure 4F). Together, these data provide corroborative evidence that $\alpha 2 \delta 2$ pharmacological blockade through GBP administration aids structural and functional reorganization of the corticospinal tract after SCI.

Adult mice receiving GBP recover forelimb function 4 months after cervical SCI. Next, we tested whether corticospinal reorganization promotes recovery of forelimb function after cervical SCI. We first confirmed that corticospinal function is required during forelimb skilled walking (4). To test this, we injected AAV particles expressing the designer receptor exclusively activated by designer drug (DREADD) hM4Di (27) into the right sensory-motor cortex to transiently silence the corticospinal pathway on 1 side of adult naive mice when clozapine $\mathrm{N}$-oxide (CNO) is administered (Supplemental Figure 7A). Corticospinal silencing resulted in impaired skilled walking on the horizontal ladder, as shown by the decrease in the percentage of correct forelimb steps (Supplemental Figure 7, B and C). We obtained similar results 7 days after unilateral PTX (Supplemental Figure 7, D and E), as reported by others (28). After collecting preoperative baseline measures, adult mice were subjected to a left C5 lateral hemisection. The mice were treated with either GBP or vehicle beginning 1 hour after injury (Figure $5 \mathrm{~A}$ ). Whereas mice treated with the vehicle demonstrated correct forelimb placement in only $30.58 \pm 3.76 \%$ of their steps at 131 days after injury, GBP-treated mice showed correct placement in $58.91 \pm 3.69 \%$ of their steps (Figure 5B and Supplemental Video 1). These results were replicated in a separate cohort of mice (Figure 5C). No difference was found in hindlimb correct placement (Supplemen- 
A

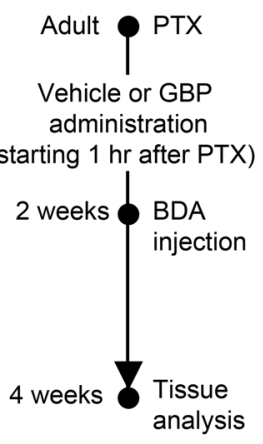

C

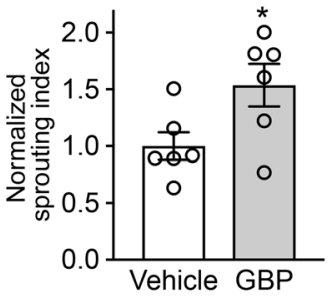

D

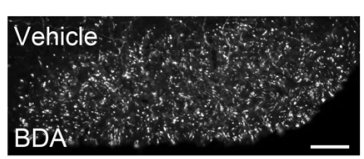

GBP

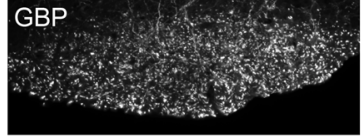

B
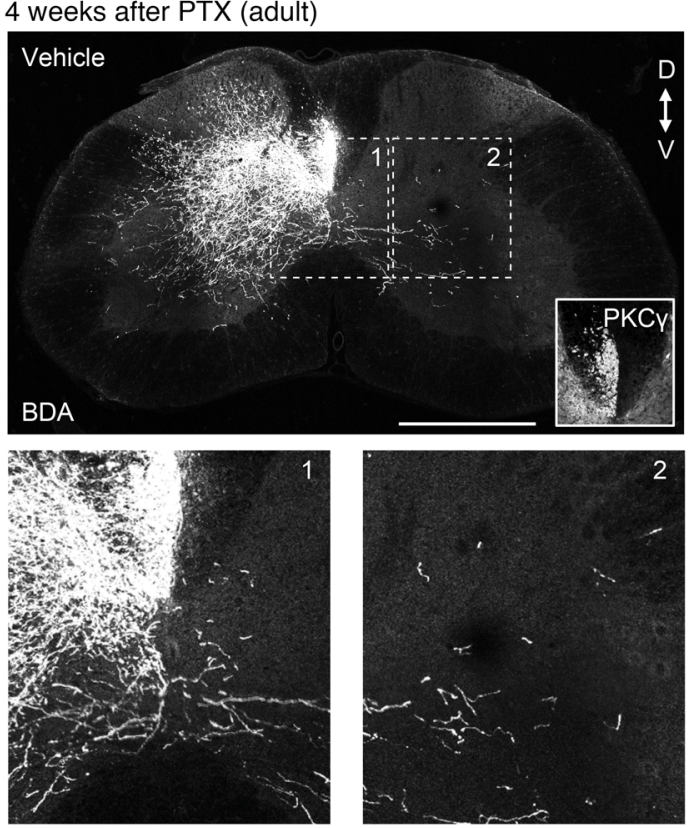

$\mathbf{E}$

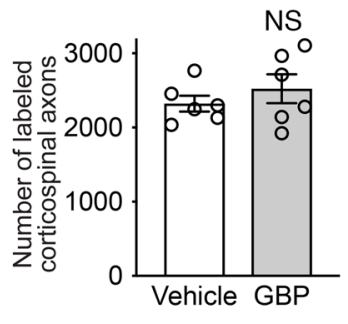

$\mathbf{F}$

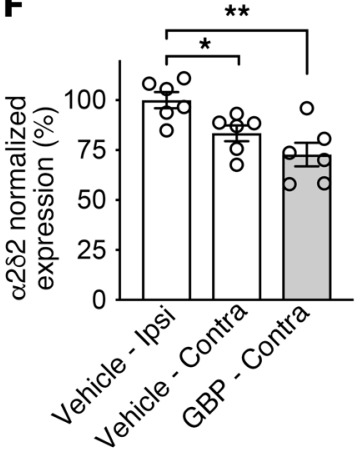

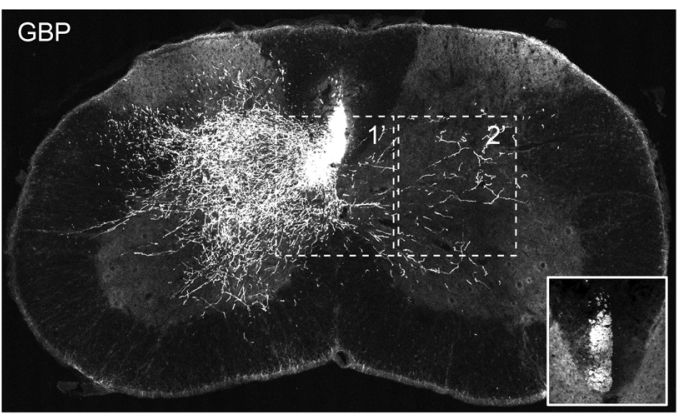
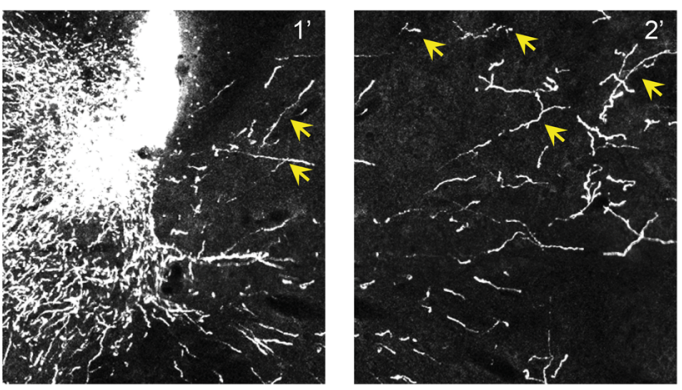

G
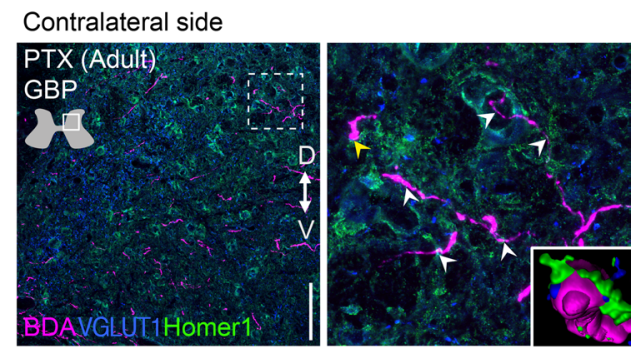

Figure 3. $\boldsymbol{\alpha 2} \delta \mathbf{2}$ pharmacological blockade promotes corticospinal sprouting in adulthood. (A) Experimental scheme of B. (B) Representative fluorescence images of C7 spinal cord sections from adult mice with unilateral PTX performed at 8 weeks of age. PKC $\gamma$ staining is shown to confirm lesion completeness. The arrows indicate sprouting corticospinal axons (bottom panels). Scale bar: $500 \mu \mathrm{m}$. D, dorsal; V, ventral. (C) Quantification of B. Mean and SEM (unpaired 2-tailed Student's $t$ test ${ }^{*} P<0.05$; vehicle $n=6$ and GBP $n=6$ mice). (D) Coronal sections of the medullary pyramid showing BDA-labeled corticospinal axons. Scale bar: $50 \mu \mathrm{m}$. (E) Quantification of $\mathbf{D}$ (unpaired 2-tailed Student's $t$ test NS, not significant; vehicle $n=6$ and GBP $n=6$ mice). (F) $\alpha 2 \delta 2$ expression in corticospinal neurons 4 weeks after PTX. Sagittal sections of the mouse brain (left and right hemispheres) were immunostained with $\alpha 2 \delta 2$ antibody. Mean and SEM (1-way ANOVA followed by Dunnett post test ${ }^{*} P<0.05$; ${ }^{*} P<0.01$; vehicle $n=6$ and GBP $n=6$ mice, $349-396$ neurons per condition). (G) Representative fluorescence images of C7 spinal cord sections from mice with unilateral PTX performed in adulthood treated with GBP. The arrows indicate excitatory synaptic puncta along sprouting corticospinal axons. Inset, $3 \mathrm{D}$ reconstruction of the region in the main panel indicated by the yellow arrow. Scale bar: $100 \mu \mathrm{m}$.

tal Figure 8A). GBP ameliorated mechanical hypersensitivity and partially restored forelimb symmetry in rearing after SCI (Supplemental Figure 8, B and C).

We measured the forelimb toe spread as an indicator of muscle function and found increased toe spread on the ipsilateral forepaw in mice administered GBP ( $n=7$ of 9) (Figure 5, D and E). Moreover, we recorded open-field spontaneous activity and discovered that GBP-treated mice spent more time resting than vehicle-treated mice (Supplemental Figure 8, D and E). No differences were found in the total distance traveled and vertical episode counts (Supplemental Figure 8D). Three months after SCI, we injected AAV particles expressing the DREADD receptor hM4Di into the right forelimb sensory-motor cortex to chemogenetically silence corticospinal projections and determine the net contribution of corticospinal reorganization to any observed behavioral recovery (Figure 5F). Chemogenetic silencing of ipsilateral corticospinal projections abolished the functional recovery in GBP-treated mice 4 months after SCI, as shown by the decrease in the percentage of correct forelimb steps and forelimb symmetry in rearing (Figure $5, \mathrm{G}$ and $\mathrm{H}$ ). Collectively, these data provide direct evidence that GBP-mediated reorganization of the corticospinal pathway promotes recovery of forelimb function after cervical SCI.

\section{Discussion}

Our study indicates that $\alpha 2 \delta 2$ pharmacological blockade through GBP enables adult corticospinal neurons to undergo structural and 
functional changes after CNS injury, effectively promoting recovery of forelimb function after cervical SCI. Hence, corticospinal sprouting and regeneration increased in injured mice receiving GBP. Together with our previous findings in dorsal root ganglia neurons (17), these results underscore a critical role of $\alpha 2 \delta 2$ in restraining axon growth and regeneration in mammalian CNS neurons. Upregulation of $\alpha 2 \delta 2$ expression parallels the increased spontaneous firing of corticospinal neurons at times when the corticospinal axon growth program is near completion and synaptogenesis starts (29, 30). The design of the electrode arrays enabled us to record neuronal firing within cortical layer V, but neurons other than corticospinal neurons may also be recorded. Given that excitatory pyramidal neurons represent the majority of the neurons within layer $V$ and that interneurons represent only a small fraction (31), it is unlikely that the developmental-dependent increase in neuronal firing within cortical layer $\mathrm{V}$ predominantly originates from interneurons. If the corticospinal pathway is damaged when $\alpha 2 \delta 2$ expression in corticospinal neurons is rather low, numerous corticospinal axons from the contralateral uninjured side sprout and form new synapses into the denervated side of the cervical spinal cord. Under such experimental conditions, the $\alpha 2 \delta 2$ developmental-dependent increase in corticospinal neurons is prevented. Thus, loss of neuronal intrinsic axon growth ability may be abrogated or delayed by manipulating a developmental switch centered on $\alpha 2 \delta 2$. In the adult mouse, $\alpha 2 \delta 2$ pharmacological blockade is sufficient to promote structural corticospinal plasticity. Of note, these sprouting axons are capable of forming new synaptic contacts at correct locations within the denervated side of the spinal cord.

The expression of $\alpha 2 \delta 2$ increases in corticospinal neurons during brain development and after a cervical SCI that completely transects corticospinal axons. After SCI, lack of afferent input to the somatosensory cortex causes changes in corticospinal neurons' homeostatic synaptic plasticity that lead to cortical hyperexcitability $(23,32)$. Given that $\alpha 2 \delta 2$ positively regulates synaptic transmission (33), increased $\alpha 2 \delta 2$ expression likely contributes to maladaptive plasticity and detrimental alteration of neuronal function under several pathological conditions. Thus, $\alpha 2 \delta 2$ pharmacological blockade through early GBP administration after CNS injury may be key in shifting the balance from maladaptive to adaptive responses in injured corticospinal neurons. Further dissection of the mechanisms underlying changes in $\alpha 2 \delta 2$ expression will be an important direction for future investigations.

In cultured embryonic mouse cortical neurons, $\alpha 2 \delta 2$ is expressed at the soma, along the axon, and at the growth cone. When cultured in the presence of GBP, these neurons extend longer neurites compared with the control. GBP binds with high affinity and selectivity to both $\alpha 2 \delta 1$ and $\alpha 2 \delta 2(16,34)$. Our data, however, suggest that $\alpha 2 \delta 2$ binding is necessary for GBPmediated neurite outgrowth. The punctate distribution of $\alpha 2 \delta 2$ at the growth cone may serve to regulate cytoskeletal elements and membrane dynamics to control axon elongation. $\mathrm{Ca}^{2+}$ is known to influence growth cone motility through remodeling of the actin cytoskeleton (35-38). Extracellular guidance cues including proteoglycans, cell adhesion molecules, and myelinassociated proteins use $\mathrm{Ca}^{2+}$ to transduce cell surface signals that affect growth cone exploratory behavior (39-41). Elevated actin turnover has been recently shown to fuel axon regeneration (42).
Whether $\alpha 2 \delta 2$ pharmacological blockade alters cytoskeletal and membrane dynamics remains to be explored.

One of the first steps in rebuilding neuronal circuits after CNS trauma is the promotion of structural plasticity and axon regeneration. After an incomplete SCI, multiple forms of axon growth and regeneration exist $(26,43,44)$. Regenerating axons can extend across the lesion or around it through spared tissue (45). Yet, functional regeneration of the corticospinal tract does not proceed across the lesion in GBP-treated mice. Whereas corticospinal axons regenerate through the lesion, no difference in light-evoked LFPs in vivo was observed between the 2 experimental conditions. Therefore, regenerating corticospinal axons that cross the lesion may not be integrated into spinal circuits or simply may not be sufficient to drive changes in network activity below the lesion. Instead, functionally relevant corticospinal regeneration proceeds toward the contralateral side in mice with SCI administered GBP. These regenerating axons establish functional connections, as shown by the large LFPs recorded rostral to the site of injury. Topographic mapping of corticospinal connectivity within the $\mathrm{C} 2-\mathrm{C} 4$ region of the spinal cord highlights preferential innervation of sensory domains within the spinal gray matter. In this context, recent studies demonstrated that corticospinal projections are capable of gating sensory information through the spinal cord (46) and that excitatory VGLUT3 ${ }^{+}$interneurons located in the dorsal horns receive inputs from the corticospinal tract (47). How such selection preference is established after SCI is currently not known. Competitive interactions exist between primary sensory afferents and corticospinal axons, and these interactions are thought to contribute to the organization of spinal motor circuits (48). Accordingly, corticospinal reorganization in GBP-treated mice may develop at the expense of maladaptive plasticity of primary sensory afferents. Whether corticospinal neurons projecting within sensory domains of the spinal cord are better at regenerating and more likely to respond to our treatment strategy awaits clarification.

We show that reestablishing corticospinal connectivity within the injured spinal cord aids recovery of forelimb functions 4 months after cervical SCI. Using noninvasive chemogenetics tools (49), we demonstrate that transient silencing of injured corticospinal projections abolished recovery in GBP-treated mice 4 months after SCI. Together with antidepressants and serotonin noradrenaline reuptake inhibitors, gabapentinoids represent a first-line treatment to manage neuropathic pain conditions (50). Of note, amelioration of mechanical hypersensitivity in GBP-treated mice proceeds quickly within 1 week after SCI. However, recovery of motor function takes much longer. Therefore, it is less likely that motor recovery relies on the resolution of neuropathic pain, as shown by others (51). Based on previous findings using experimental models of neuropathic pain (52-54), we speculate that $\alpha 2 \delta 1$ binding may be responsible for GBP's rapid effect on hypersensitivity. In contrast, our results indicate that GBP-mediated reorganization of the corticospinal pathway may require binding to $\alpha 2 \delta 2$. The initiation of GBP administration is the key to maximizing any chance of functional recovery after CNS trauma. Our previous findings suggest that the maximal regenerative outcome is achieved when gabapentinoid treatment is delivered soon, rather than weeks, after SCI (17). Similarly, a multicenter cohort study has found that early (versus late) administration of gabapentinoids improves motor recovery in individuals with SCI (51). We are now poised to test the effects of 
A

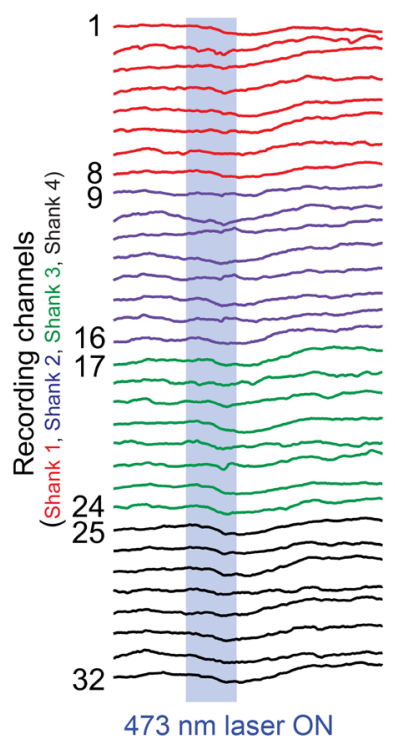

B

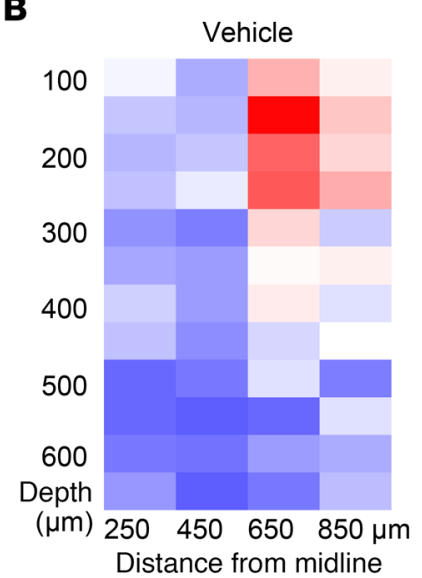

$\mathbf{E}$

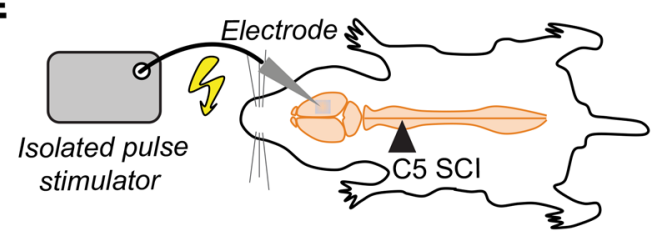

$\mathbf{F}$

c-Fos activity mapping (C2-C4 spinal cord)

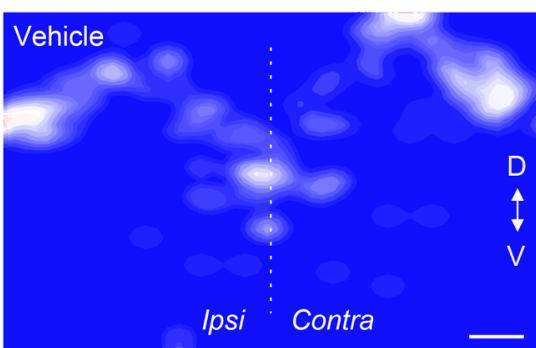

C
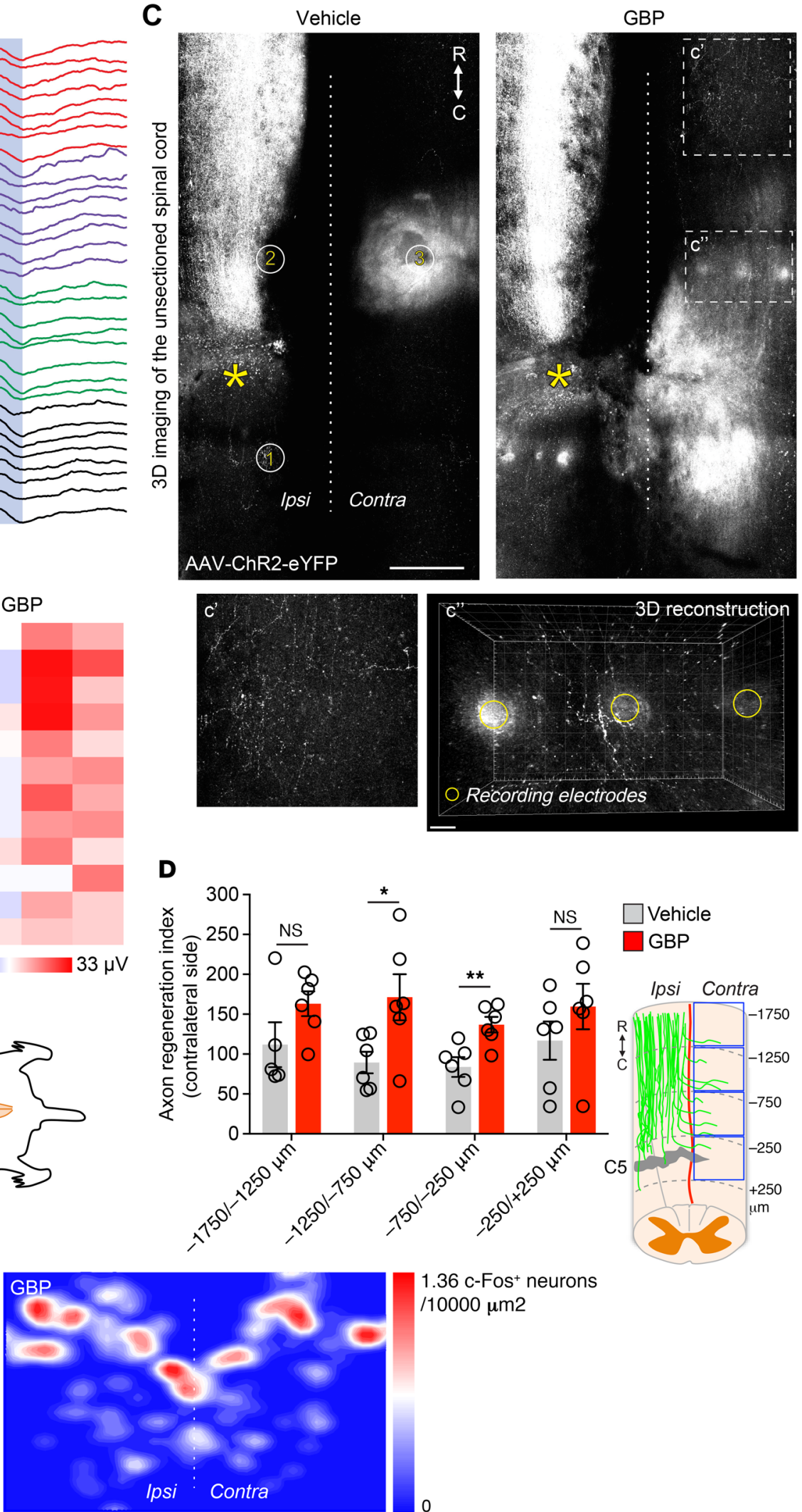
Figure 4. $\alpha 2 \delta 2$ pharmacological blockade through CBP administration promotes functionally relevant corticospinal regeneration. (A) In vivo recording of light-evoked LFPs using a 32-channel electrode array (vehicle $n=6$ and GBP $n=6$ mice). The LFP color code represents the 4 electrode shanks (B) Heatmap (z-transformed, blue to red) from $\mathbf{A}$ to visualize changes in corticospinal connectivity in the injured spinal cord. Each box represents averaged data from a single electrode, each column a single electrode shank (vehicle $n=6$ and GBP $n=6$ mice). (C) Automated tile scanning of the unsectioned adult spinal cord 4 months after $\mathrm{C5} \mathrm{SCI}$. Corticospinal axons were labeled by injecting AAV-ChR2-eYFP into the right sensory-motor cortex. The asterisk indicates the lesion epicenter. Enclosed numbers indicate the location where multichannel electrodes arrays were inserted for in vivo recording. Scale bar: $500 \mu \mathrm{m} ; 50 \mu \mathrm{m}$ (inset). R, rostral; C, caudal. (D) Quantification of C. Mean and SEM (mixed model with repeated measures using unstructured covariance matrix, ${ }^{*} P<0.05$; ${ }^{* *} P<0.01$; NS, not significant; vehicle $n=6$ and GBP $n=6$ mice). (E) Schematic of the cortical stimulation paradigm. (F) c-Fos activity mapping in the injured spinal cord (vehicle $n=6$ and GBP $n=5$ mice). Scale bar: $200 \mu \mathrm{m}$. D, dorsal; V, ventral.

delaying the treatment 1-7 days after spinal cord contusion to determine the translational relevance of our pharmacological strategy and to address a recurrent problem related to actively recruiting volunteers with SCI to research trials. In humans, gabapentinoids are associated with a number of adverse effects, including dizziness, drowsiness, and weight gain (55). In turn, it will be important to determine when medication may be gradually discontinued without compromising any beneficial functional outcome.

Whether our treatment strategy may synergize in experimental models of chronic SCI with other promising interventions, such as administration of the membrane-permeable intracellular sigma peptide that binds and inactivates protein tyrosine phosphatase sigma $(56,57)$, or exercise and cardiovascular training $(58,59)$ remains to be tested. Whereas rehabilitative training allows refining and stabilizing newly formed neuronal networks, intensive training early after injury negatively affects network reorganization, leading to recovery failure (60). We discovered that GBP-treated mice rest in an open field more than controls. This may not be a surprise given that $\alpha 2 \delta 2$ pharmacological blockade dampens excitatory synaptic transmission $(17,61,62)$, but the contribution of rest to the recovery process with or without rehabilitation after SCI deserves attention for future investigation.

Altogether, our results present a strong inducement for further exploring $\alpha 2 \delta 2$ pharmacological blockade through gabapentinoid administration as a practical and promising clinical strategy for addressing CNS repair after SCI.

\section{Methods}

\section{Animals}

Adult (7- to 8-week-old) female and male C57BL/6J mice (stock no. 000664, The Jackson Laboratory) were used for all experiments, except those specifying different developmental stages and GFP-M mice. GFP-M (stock no. 007788; RRID:IMSR_JAX 007788) mice expressing GFP under the control of Thy1 promoter were purchased from The Jackson Laboratory. Mice were randomly assigned to experimental groups. Experimenters were blind to group assignment and experimental conditions.

\section{Antibodies}

The following antibodies were used: mouse monoclonal anti- $\beta$ III tubulin (Tuj1) (801202, RRID:AB_10063408, BioLegend), rabbit polyclonal anti- $\beta$ III tubulin (T2200, RRID:AB_262133, Sigma-Aldrich), mouse monoclonal anti-NeuN (MAB377, RRID:AB_2298772, Millipore), Alexa Fluor 488 Phalloidin (A12379, RRID:AB_2315147, Invitrogen), rabbit polyclonal anti- $\alpha 2 \delta 2$ (ACC-102, RRID:AB_11124467, Alomone Labs), rabbit monoclonal anti-c-Fos (9F6) (2250S, RRID:AB_2247211, Cell Signaling Technology), rabbit polyclonal anti-GFAP (Z0334, RRID:AB_10013382, Dako), rabbit polyclonal anti-mCherry (ab167453, RRID: AB_2571870, Abcam), rabbit monoclonal anti-PKC gamma (59090, RRID: AB_2799557, Cell Signaling Technology), chicken polyclonal anti-Homer1 (160006, RRID:AB_2631222, Synaptic Systems), guinea pig polyclonal anti-VGLUT1 (135304, RRID:AB_887878, Synaptic Systems), rabbit polyclonal anti-Caspase-3 (ab 13847, RRID AB_443014, Abcam), and chicken polyclonal anti-GFP (GFP-1020, RRID:AB_10000240, Aves Labs).

\section{Primary neuronal cultures}

Cortical neuronal cultures were derived from cortices of embryonic 17.5-day-old mouse (C57BL/6J) embryos. Cortices were extracted, dissociated, and cultured as previously reported (63). Briefly, cortices were minced and dissociated in the same buffer with $1,800 \mathrm{U} / \mathrm{ml}$ tryp$\sin$ at $36.5^{\circ} \mathrm{C}$ for 20 minutes. Next, $200 \mathrm{U} / \mathrm{ml}$ DNase I and 3,600U/ $\mathrm{ml}$ soybean trypsin inhibitor were added to the suspension, and cells were triturated through a $5-\mathrm{ml}$ pipette. The tissue was allowed to settle for 5-10 minutes, and then the supernatant was collected, and the remaining tissue pellet was retriturated. The combined supernatants were centrifuged through a 4\% BSA (A3059, Sigma-Aldrich) layer and the cell pellet was resuspended in neuronal seeding medium (NSM), which consisted of Neurobasal Medium (12348017, Life Technologies) supplemented with B27 (17504044, Life Technologies). The culture was maintained in a humidified atmosphere containing $5 \% \mathrm{CO}_{2}$ in air at $36.5^{\circ} \mathrm{C}$. To achieve Cacna $2 \mathrm{~d} 2$ overexpression, dissociated cortical neurons were electroporated (program: CA138, NC0301987, Fisher Scientific) with a mixture of GFP ( $2.5 \mu \mathrm{g}$, pmaxGFP, Lonza) plus either Cacna2d2 (4 $\mu \mathrm{g}, \mathrm{MC} 223740$, Origene), Cacna2d2(R282A) (17) or empty $(4 \mu \mathrm{g})$ plasmid DNA. Electroporated neurons were then plated at low density on coverslips coated with poly-D-lysine (P6407, Sigma) and the electroporation medium was replaced with fresh medium (with or without GBP) 2 hours after plating.

\section{Corticospinal neurons retrograde labeling and immunohistochemistry} A C3-5 laminectomy was performed in GFP-M adult mice, and FluoroGold tracer (1\%, Fluorochrome) was injected $(0.5 \mu \mathrm{l} /$ spot at $0.1 \mu \mathrm{l} / \mathrm{min}$, 4 spots) into the dorsal corticospinal at the C3-5 spinal cord. After 3 days, the mice were perfused and the brains dissected and sequentially dehydrated in $10 \%, 20 \%$, and $30 \%$ sucrose. Tissues were then embedded in optimum cutting temperature (OCT) compound (Tissue-Tek), frozen, sectioned (20- to 30- $\mu$ m thick, HM525 NX, Thermo Fisher Scientific) and mounted on slides. Slides were warmed at $37^{\circ} \mathrm{C}$ for 30 minutes and OCT was washed away with PBS. Sections were then blocked at room temperature with $2.5 \%$ bovine serum albumin (A3059, Sigma-Aldrich) in PBS with 0.1\% Triton-X100 for 1 hour and incubated overnight at $4^{\circ} \mathrm{C}$ with the primary antibody. After washing 3 times with PBS, sections were incubated with Alexa Fluor-conjugated secondary antibodies (1:500, Life Technologies). When necessary, sections were 
A

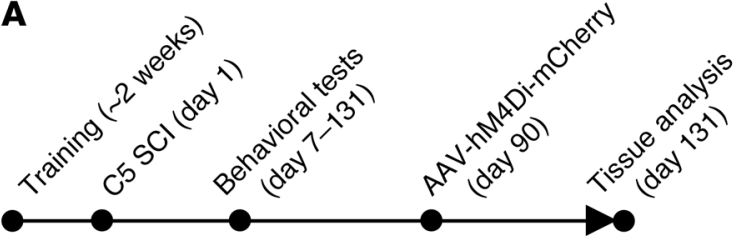

Vehicle or GBP administration (starting $1 \mathrm{hr}$ after $\mathrm{SCl}$ )

B

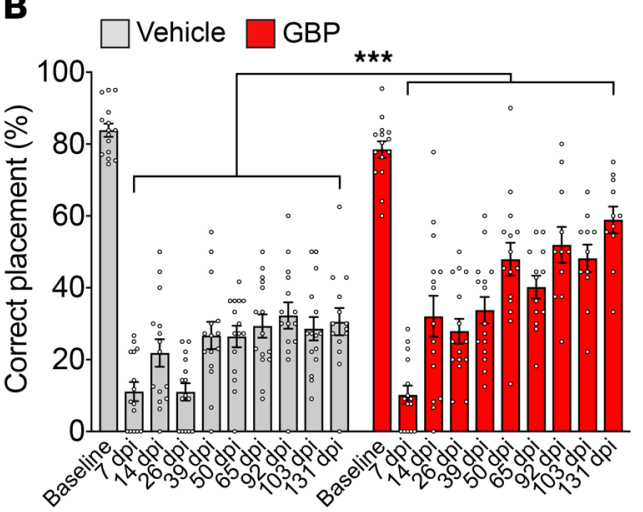

C

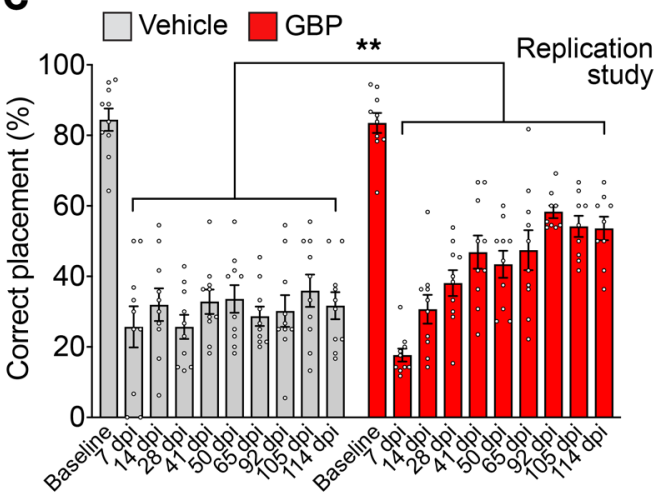

D

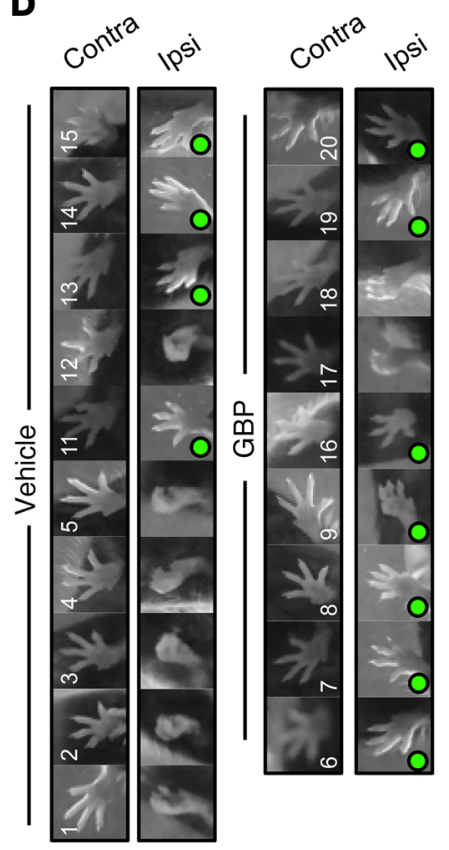

E

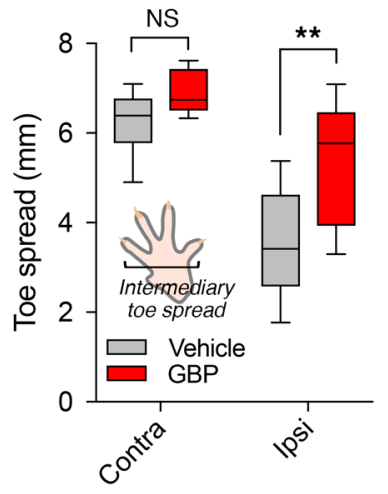

$\mathbf{F}$

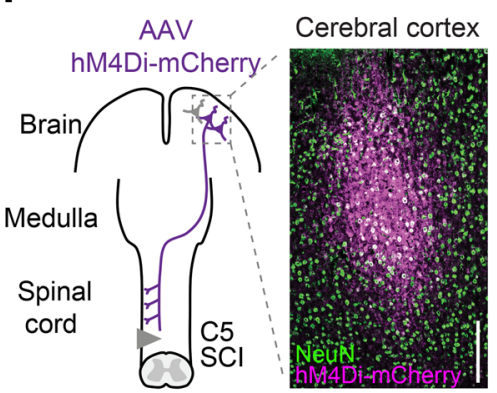

G

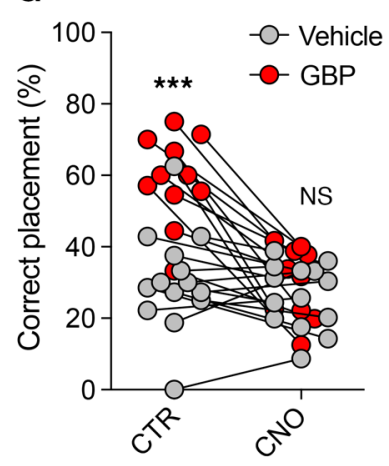

H

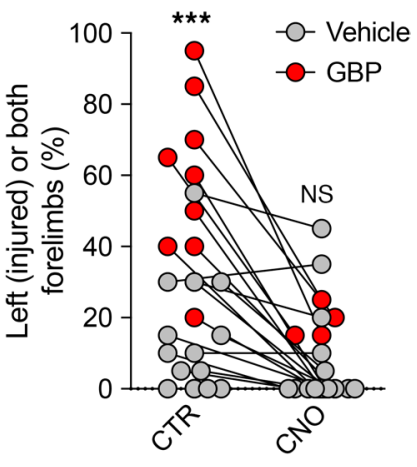

Figure 5. Mice treated with GBP recover forelimb function after cervical SCI. (A) Experimental scheme. (B) Recovery of forelimb skilled locomotor function was measured using the horizontal ladder rung-walking test. Mean and SEM (mixed model with repeated measures using compound symmetry covariance structure and controlled on baseline values ${ }^{* * *} P<0.001$; vehicle $n=15$ and GBP $n=15$ mice). (C) Replication of the study shown in B. Mean and SEM (mixed model with repeated measures using compound symmetry covariance structure and controlled on baseline values, ${ }^{* *} P<0.01$; vehicle $n=10$ and GBP $n=10$ mice). (D) Photographs showing forelimb footprints used for intermediary toe spread measurements. Green circles indicate normal toe spread. (E) Quantification of D. Box and whiskers (minimum to maximum) (2-way ANOVA followed by Tukey's post test ${ }^{*} P<0.001$; NS, not significant; vehicle $n=10$ and CBP $n=9$ mice). (F) Coronal sections of the brain were stained using NeuN and mCherry antibodies. Scale bar: $200 \mu \mathrm{m}$. Transient activation of hM4Di in corticospinal neurons abrogates recovery of (C) forelimb skilled walking and (H) forelimb symmetry (cylinder test) in rearing after $\mathrm{C5} \mathrm{SCl}$ in mice receiving GBP. Scatter plot (2-way ANOVA followed by Bonferroni's post test ${ }^{* *} P<0.001$; NS, not significant; vehicle $n=14$ and GBP $n=11$ mice). counterstained with DAPI (1:10,000, D9542, Sigma-Aldrich). Images were taken using a confocal (SP8, Leica and C2 plus, Nikon) or epifluorescence microscope (Axio Observer Z1, Zeiss) and linear fluorescence intensity was calculated using Photoshop (version 20.0.1, Adobe) or ImageJ after background subtraction. The expression of $\alpha 2 \delta 2$ (1:400) in Fluoro-Gold-labeled corticospinal neurons was measured by using Zen Blue software (Zeiss). A minimum of 3 independent biological replicates ( 2 or more sections/mouse) was analyzed per condition.

\section{Immunoblot analysis}

For immunoblotting, cultured embryonic cortical neurons and dissected sensory-motor cortices from mice at different stages of postnatal development were each lysed on ice in RIPA buffer (500 mM Tris- $\mathrm{HCl} \mathrm{pH} \mathrm{4.8,} 150 \mathrm{mM} \mathrm{NaCl}, 1 \%$ Triton, 0.5\% sodium deoxycholate, $0.1 \%$ sodium dodecyl sulfate) containing phosphatase (04906845001, Sigma-Aldrich) and protease inhibitors (5892791001, Sigma-Aldrich), centrifuged, and the supernatant 
collected. Using Bradford reagent (Bio-Rad), the protein concentration of the lysate was determined, and a portion of the lysate (10-20 $\mu \mathrm{g}$ total protein) was then fractionated by SDS-polyacrylamide gel electrophoresis (PAGE). The separated proteins were transferred to a nitrocellulose membrane $(0.2 \mu \mathrm{m}$, Bio-Rad), which was stained to confirm equal loading and transfer of the samples with Ponceau $\mathrm{S}$ (P7170, MilliporeSigma). After blocking at room temperature with $5 \%$ nonfat milk (170-6,404, Bio-Rad) in TBST for 1 hour, the membrane was then probed with a rabbit polyclonal anti- $\alpha 2 \delta 2$ (1:1,000). Rabbit polyclonal anti-Tuj1 (1:20,000, Sigma-Aldrich) antibody was used as protein-loading controls. Densitometry analysis was done using Image J (NIH). The regions of interest that contained the bands to quantify had the same size across the immunoblot. After background subtraction, the intensity of $\alpha 2 \delta 2$ bands at 130 and $105 \mathrm{kDa}$ was measured and normalized to the loading control (e.g., Tuj1). To calculate $\alpha 2 \delta 2$ expression, the 2 values were then summed. Three biological replicates for each experimental condition were analyzed.

\section{RNA isolation}

Total RNA was extracted from mouse cortices using the RNeasy kit (Qiagen) and cDNA was synthesized from 0.1-0.5 $\mu \mathrm{g}$ of RNA using random hexamers from the SuperScript VILO cDNA synthesis kit (11754050, Thermo Fisher Scientific). The subsequent cDNA was used in a real-time PCR (StepOne Plus, Applied Biosystem) using Fast SYBR Green Master Mix (4385612, Applied Biosystem). Melting curve reactions were run with each primer set. The $\beta$ actin gene was used for normalization. The sequences of the primers used were as follows: Cacna2d2_s 5'-ACAAGGACAATCGGAACCTG-3', Cacna2d2_as 5'-TGGGCTTTCTGGAAATTCTCT-3', $\beta$ actin_s 5'-ACAGCTTCACCACCACAGCTGA- $3^{\prime}, \beta$ actin_as $5^{\prime}$-GAGGTCTTTACGGATGTCAACGTC-3'. Normalized expression was calculated as dCt (gene norm) $=\mathrm{Ct}$ Cacna2 $\mathrm{d} 2-\mathrm{Ct} \beta$ actin and normalized expression $={ }^{2}-\mathrm{dCt}$ (gene norm)

\section{In vivo recording of spontaneous firing}

P14 and P28 mice were anesthetized with a mixture of ketamine (100 $\mathrm{mg} / \mathrm{kg}$ body weight) and xylazine (10 $\mathrm{mg} / \mathrm{kg}$ body weight), and P7 with a mixture of ketamine $(50 \mathrm{mg} / \mathrm{kg}$ body weight) and xylazine ( $5 \mathrm{mg} / \mathrm{kg}$ body weight). A craniotomy was performed to expose the right sensory-motor cortex. A 32-channel silicon electrode array (Buzsaki 32-A32, NeuroNexus Technologies) connected to a stereotaxic frame was inserted 500 to $600 \mu \mathrm{m}$ deep into the forelimb sensory-motor cortex. Spontaneous neuronal firing was recorded at a $25-\mathrm{kHz}$ sampling rate and low-pass filtered at $10 \mathrm{kHz}$ using the SmartBox acquisition system (NeuroNexus Technologies). Recording data were analyzed using Igor Pro (version 8, WaveMetrics). Briefly, data were filtered off-line at 300 to $3,000 \mathrm{~Hz}$ and smoothed using a sliding average of 5 points. Spikes were identified when passing the detection threshold of 4 times the baseline noise standard deviation (64-66). Detected spikes were then sorted into single units using the k-means clustering method after the principal component analysis $(64,66)$. The customized algorithm was scripted in Igor Pro and the experimenter manually reviewed sorted results.

\section{Cervical spinal cord injury and corticospinal transduction}

Mice were anesthetized with a mixture of ketamine $(100 \mathrm{mg} / \mathrm{kg}$ body weight) and xylazine (10 mg/kg body weight). A C5 laminectomy was performed, and the spinal cord was crushed with no. 5 modified forceps (11254-20, Dumont, FST). The forceps were positioned to completely sever half of the spinal cord (Supplemental Figure 1D). Beginning 1 hour after injury, GBP ( $46 \mathrm{mg} / \mathrm{kg}$ body weight, PHR1049, CAS:60142-96-3, MilliporeSigma) or the corresponding volume of vehicle (0.9\% sodium chloride, 0409-7138-09, Aqualite system) was administered (intraperitoneal injections, 3 times/day for the first week, 2 times/day until the end of the study). Three to four weeks before the end of the study, AAV particles for optogenetic or chemogenetic manipulation of neurons were injected into the right sensory-motor cortex (AP coordinates from bregma in $\mathrm{mm}$ : AP 1.0/1.3, 0.5/1.3, 0/1.3, -0.5/1.3, all at a depth of $0.6 \mathrm{~mm}$ from the surface, $500 \mathrm{nl} /$ injection site). For immediate-early gene mapping, a cohort of mice with SCI was anesthetized, and electrical stimulation $(300 \mu \mathrm{A}, 0.5-\mathrm{ms}$ biphasic pulse at $5 \mathrm{~Hz}$ for 15 minutes) of the right sensory-motor cortex was delivered using a tungsten concentric bipolar electrode (TM33CCINS, World Precision Instruments) connected to an isolated pulse stimulator (A-M systems Model 2100). The electrode was inserted at a depth of $0.5 \mathrm{~mm}$ from the surface (AP coordinates from bregma in mm: AP 0.25/1.3). At the end of each study (1 hour after stimulation for early gene mapping), mice were perfused with $4 \%$ paraformaldehyde. Sagittal $(60-\mu \mathrm{m}$ thick) and transverse (20- to $30-\mu \mathrm{m}$ thick; $2.5 \mathrm{~mm}$ rostral and 6-7 $\mathrm{mm}$ caudal to the lesion) serial cryosections of the spinal cord were cut and collected to confirm the completeness of the lesion and tracing efficiency among experimental groups. Mice with incomplete lesions were excluded from the analysis. In mice injected with AAV-ChR2-eYFP, the portions of the unsectioned spinal cords containing the lesion site were cleared using the advanced CUBIC protocol (67) and imaged using a confocal microscope (SP8, Leica). The imaging software Imaris (version 9.2, Bitplane) was used for 3-dimensional rendering (Figure $4 \mathrm{C}$ ). Three-dimensional images were used for quantification of regeneration using the ImageJ Simple Neurite Tracer plugin (68). Briefly, the contralateral side of the injured spinal cord was divided in 4 adjacent boxes $(-1750 /-1250$ $\mu \mathrm{m},-1250 /-750 \mu \mathrm{m},-750 /-250 \mu \mathrm{m}$, and $-250 /+250 \mu \mathrm{m}$ from the lesion epicenter), each measuring $1,000 \mu \mathrm{m}$ in width and $500 \mu \mathrm{m}$ in length, and the ipsilateral side only contained 1 measuring box with the same size immediately below the lesion epicenter $(0 /+500$ $\mu \mathrm{m})$. Regenerating axons were then semi-automatically traced in each 3-dimensional box. For the contralateral side, the total length was calculated and divided by the number of corticospinal fibers counted at the level of medulla oblongata (c.a., $1 \mathrm{~mm}$ proximal to the pyramidal decussation) to account for variation in tracing efficiency in each mouse. The procedure to count corticospinal fibers at the medullary level was the same as described in Liu et al. (11). The axon regeneration values at different distances were displayed as a percentage of the average value of the vehicle group in all 4 boxes (Figure 4D). For the ipsilateral side, we calculated the number of traced corticospinal axons below the lesion site and normalized it to the number of labeled corticospinal axons at the medullary level. Immunohistochemistry was performed following standard protocols (see above, $\alpha 2 \delta 2$ 1:400, NeuN 1: 500, GFAP 1: 500, mCherry 1:300, GFP 1:500, PKC 1 1:200). Immunohistochemistry for c-Fos (1:1,000, Cell Signaling Technologies) required an antigen-retrieval procedure consisting of 2-3 minutes incubation of tissue slices in citric acid-based antigen-unmasking solution $\left(\mathrm{H}-3300\right.$, Vector Laboratories) at $95^{\circ} \mathrm{C}-100^{\circ} \mathrm{C}$. The average den- 
sity of c-Fos/NeuN positive cells within the spinal cord was then mapped using a 2-dimensional histogram. Standardized randomization and blinding strategies were adopted.

\section{PTX}

Adult mice were anesthetized with a mixture of ketamine $(100 \mathrm{mg} /$ $\mathrm{kg}$ body weight) and xylazine (10 $\mathrm{mg} / \mathrm{kg}$ body weight); at P10, they were anesthetized with a mixture of ketamine $(50 \mathrm{mg} / \mathrm{kg}$ body weight) and xylazine ( $5 \mathrm{mg} / \mathrm{kg}$ body weight). An incision was made at the left side of the trachea and a blunt dissection exposed the base of the skull where, to access the medullary pyramids, a craniotomy in the occipital bone was performed. The left pyramid was cut with a micro scalpel (no. 715, Feather) medially to the basilar artery and the wound was closed in layers with 6.0 (adult) and 9.0 (P10) sutures. The sham operation for the P10 cohort (sham and PTX surgeries were performed at P10) included a craniotomy in the occipital bone without cutting the left pyramid. The mice were placed on soft bedding in their home cage on a warming surface held at $37^{\circ} \mathrm{C}$ until awake and alert. After 2 weeks (PTX in adulthood) or 18 days (PTX at P10), the intact corticospinal tract was traced with $10 \%$ BDA (10,000 MW, D1956, Life Technologies). The mice were perfused with $4 \%$ paraformaldehyde 2 weeks after BDA injection. Spinal cords and medulla oblongata were post-fixed in $4 \%$ paraformaldehyde overnight at $4^{\circ} \mathrm{C}$ and then immersed in 10\%, 20\%, and 30\% sucrose, and cryosections were prepared. After quenching endogenous peroxidase with $0.3 \%$ $\mathrm{H}_{2} \mathrm{O}_{2}$ in PBS for 30 minutes, coronal sections were incubated for 2 hours with streptavidin-horseradish peroxidase conjugate (1:200 in 2\% Triton PBS, NEL7500001EA, Perkin Elmer). The TSA Cyanine 3 system (SAT704A001EA, Perkin Elmer) was then used for immunofluorescence amplification of the BDA signal. The procedure to count corticospinal fibers at the medullary level was the same as described above. For quantification of sprouting corticospinal axons, the area of sprouting axons from the contralateral side was measured and divided by the total area of gray matter from the contralateral side (the detection threshold was set as 2 times the gray matter background signal of each individual image using ImageJ). This value was then normalized to the average value from the sham control (PTX at P10) or vehicle (PTX in adulthood) groups and presented as a sprouting index. Immunostaining was performed following standard procedure ( $\mathrm{PKC} \gamma$ 1:200, NeuN 1:500, VGLUT1 1:1000, Homer1 1: 400, Caspase-3 1:300). Multidimensional surface reconstruction of putative synapses along BDA-labeled corticospinal axons was created using an Image $3 \mathrm{D}$ viewer plugin (69). The imaging stack was filtered through a $2 \mathrm{D}$ Gaussian filter (radius: 2 pixels) to reduce signal noise. Surface reconstruction was then created for each channel. The image was then rotated to confirm presynaptic and postsynaptic alignment.

\section{Immunocytochemistry and morphometric analysis}

Mouse cortical neurons were grown on PDL-coated coverslips and fixed with $4 \%$ paraformaldehyde in $4 \%$ sucrose. Coverslips were then blocked at room temperature for 1 hour with $2.5 \%$ BSA and $0.1 \%$ Triton-X100 in PBS and incubated at $4^{\circ} \mathrm{C}$ overnight with the appropriate primary antibodies (Tuj1 1:1,000, Phalloidin 1:200, and $\alpha 2 \delta 2$ 1:400). After 3 rinses in PBS, the coverslips were incubated with Alexa Fluor-conjugated secondary antibodies (1:500, Life Technologies) and washed in PBS before mounting onto microscope slides. Fluorescence images were randomly taken with an inverted microscope (Axio Observer Z1, Zeiss) and ana- lyzed using Zen Blue (Zeiss) and Fiji software (version 2.0.0-rc-54/1.5h) (70). This process was repeated for at least 3 independent experiments. The number of neurons quantified for each condition is indicated in the corresponding figure legend.

\section{Optogenetics and in vivo multichannel recording of LFPs}

AAV2/1-CamKIIa-hChR2(H134R)-eYFP (1-2 $\mathrm{e}^{13} \quad \mathrm{GC} / \mathrm{ml}$, Addgene26969, Addgene) particles were injected into the right sensory-motor cortex. Four weeks after AAV injection, the mice were anesthetized and a laminectomy was performed to expose the cervical spinal cord between C3 and C6. The vertebral columns were stabilized by clamps attached to either side of the laminectomy site. A 32-channel silicon electrode (A4x8-5mm-50-200-177, NeuroNexus Technologies) connected to a stereotaxic frame was inserted at a depth of 500 and $700 \mu \mathrm{m}$ into the spinal cord at the following locations: $500 \mu \mathrm{m}$ rostral/ipsilateral, $500 \mu \mathrm{m}$ caudal/ipsilateral, and $500 \mu \mathrm{m}$ rostral/ contralateral to the lesion site. A 473-nm diode-pumped solid-state laser (Shanghai Laser and Optics Century) was coupled with a 200$\mu \mathrm{m}$ optical fiber, and the tip of the optical fiber was placed at c.a. $2 \mathrm{~mm}$ above the spinal cord rostral to the lesion site. Using a pulse waveform generator (Keysight, 33521B), the laser was set to deliver an approximately $25-\mathrm{mW} 10-\mathrm{ms}$ pulse every 5 seconds. The laser power was measured using a slide power sensor (S170C, Thorlabs) coupled with a laser power meter (PM100A, Thorlabs). The LFP was recorded at a $25-\mathrm{kHz}$ sampling rate and low-pass filtered at $10 \mathrm{kHz}$ using the SmartBox acquisition system (NeuroNexus Technologies). Recording data were analyzed using Igor Pro software (version 8, WaveMetrics). LFPs were low-pass filtered at $50 \mathrm{~Hz}$. For each channel, 3-5 light-induced LFPs were averaged for quantification.

\section{Chemogenetics}

For chemogenetics experiments, corticospinal axons that project to the cervical spinal cord were transduced by injecting AAV2/1-hSyn$\mathrm{hM} 4 \mathrm{D}$ (gi)-mCherry (1-2e $\mathrm{e}^{13} \mathrm{GC} / \mathrm{ml}$, Addgene50475, Addgene) into the right forelimb sensory-motor cortex. Four weeks after AAV injection, the mice underwent horizontal ladder and cylinder behavioral tests starting 15 minutes after injection of CNO (1 mg/kg, i.p.) (4936, CAS: 34233-69-7, Tocris Bioscience) to transiently silence corticospinal projections. On the following day, the mice were administered an equal amount of vehicle ( $0.9 \%$ saline), and the behavioral procedure was repeated. The alternation of $\mathrm{CNO}$ and vehicle administration was repeated for 2 consecutive sessions for 1 week, and the results were averaged between sessions (Figure 5, G and H). Behavioral analysis was carried out by investigators blinded to the treatment.

\section{Behavioral testing}

Horizontal ladder. Before injury, mice were trained to walk across the ladder to an enriched cage in 1 direction. Baseline values were collected for all mice prior to injury. After the injury, and at regular intervals until the study endpoint, mice were placed at one end of the ladder and video recorded while moving across to the other end where the enriched cage was located. The recordings were then analyzed using VLC player, and the percentage of correct steps was calculated.

Toe spread index. Contralateral and ipsilateral forepaws were photographed (Sony Cybershot DSC-W800) at $117 \mathrm{dpi}$ from the ventral surface. All images were taken when the mouse was bearing weight on a glass surface. Intermediary toe spread was established from the 
images by measuring the distance between the first and fourth toe using Image J software.

Cylinder test. The mice were placed in a 500-ml clear beaker with a small amount of bedding on the bottom. The mice were allowed to move independently and explore normally. The proportion of right versus left versus both paw placements on the side of the beaker was noted for 10 attempts and used to calculate the percentage of forelimb asymmetry. Baseline values were collected for all mice prior to injury and continued at regular intervals until the study endpoint.

Von Frey test. The mice were placed in a testing chamber, and the plantar threshold was measured using retractable monofilaments (Ugo Basile) and the "up-down" method. A quick withdrawal of the left hind paw was considered as a positive response. A pause of 20-30 seconds was given to allow sensory receptors to reach baseline levels between each monofilament application. The response threshold was calculated as the lowest force (in grams) that produced a retraction at least $50 \%$ of the time.

Activity box. The mice were placed in activity boxes (Columbus Instruments) for 10 minutes. Spontaneous activities in horizontal and vertical planes were recorded through compatible Fusion software (version 6.4 r1194, Omnitech Electronics). Baseline values were collected for all mice prior to injury and continued at regular intervals until the study endpoint.

For all behavioral tests described above, experimenters collecting and analyzing data were blinded to the treatment.

\section{Statistics}

Statistical analysis was performed using Prism (version 8.0.2; GraphPad Software) as follows: unpaired 2-tailed Student's $t$ test (Figure 1K; Figure 2, C, E, G, K, and M; Figure 3, C and E; Supplemental Figure 1F; Supplemental Figure 3B; and Supplemental Figure 5F), paired 2-tailed Student's $t$ test (Supplemental Figure 7, B and E), 1-way ANOVA followed by Dunnett post test (Figure 1F, Figure 3F, and Supplemental Figure 2B), 2-way ANOVA followed by Tukey's post test (Figure 5E), 2-way ANOVA followed by Bonferroni's post test (Figure 5, G and H). Trend tests and mixed models were performed using SAS (SAS 9.4; SAS Institute) as follows: linear trend test with $\log _{2}$ transformation (Supplemental Figure $1 \mathrm{~A}$ ) or without $\log _{2}$ transformation (Figure 1, D and H), mixed model with repeated measures using unstructured covariance matrix (Figure $4 \mathrm{D}$ ), mixed model with repeated measures using compound symmetry covariance structure and controlled on baseline values (Figure 5, B and C, and Supplemental Figure 8, A-C), and mixed model with repeated measures (Supplemental Figure 8D). For all analyses performed, significance was defined as $P<0.05$. Exact values of $n$ and definition of measures are shown in the corresponding figure legends.

\section{Study approval}

All animal experiments were performed in accordance with and with the approval of the Institutional Animal Care and Use Committee at The Ohio State University.

\section{Author contributions}

WS and AT conceived the project and designed the research study. WS, MJEL, CMK, AJA, WAS, and AT performed the research. WS, MJEL, CMK, and AT analyzed the data. JP performed statistical analysis. AT supervised the research. WS and AT wrote the paper. All authors provided feedback and contributed to editing the manuscript.

\section{Acknowledgments}

We would like to thank Anthony Brown, Michele Curcio, Elizabeth Matthews, and Min Zhou for critically reading and Faith Brennan and Manoj Gottipati for help with blinding and randomization strategies. This work was supported by the Craig H. Neilsen Foundation, Marina Romoli Association, The Ohio State University Neuroscience Research Institute (NRI), and the National Institute of Neurological Disorders (grants R01NS110681 and R21NS109787), with additional support provided by NIH grant P30 NS104177.

Address correspondence to: Wenjing Sun or Andrea Tedeschi, 460W 12th Avenue, Columbus, 43210, Ohio, USA. Phone: 614.293.2672; Email: wenjing.sun@osumc.edu (WS). Phone: 614.366.6569; Email: andrea.tedeschi@osumc.edu (AT).
1. Sekhon LH, Fehlings MG. Epidemiology, demographics, and pathophysiology of acute spinal cord injury. Spine. 2001;26(24 suppl):S2-S12.

2. Maier IC, Schwab ME. Sprouting, regeneration and circuit formation in the injured spinal cord: factors and activity. Philos Trans R Soc Lond, B, Biol Sci. 2006;361(1473):1611-1634.

3. Ramer LM, Ramer MS, Bradbury EJ. Restoring function after spinal cord injury: towards clinical translation of experimental strategies. Lancet Neurol. 2014;13(12):1241-1256.

4. Jayaprakash N, Nowak D, Eastwood E, Krueger N, Wang Z, Blackmore MG. Restoration of Direct Corticospinal Communication Across Sites of Spinal Injury [preprint]. https://doi. org/10.1101/546374. Posted on bioRxiv February 11, 2019.

5. Lemon RN. Descending pathways in motor control. Annu Rev Neurosci. 2008;31:195-218.

6. Tedeschi A, Bradke F. Spatial and temporal arrangement of neuronal intrinsic and extrinsic mechanisms controlling axon regeneration. Curr Opin Neurobiol. 2017;42:118-127.
7. Anderson MA, et al. Required growth facilitators propel axon regeneration across complete spinal cord injury. Nature. 2018;561(7723):396-400.

8. Blackmore MG, et al. Krüppel-like Factor 7 engineered for transcriptional activation promotes axon regeneration in the adult corticospinal tract. Proc Natl Acad Sci U S A. 2012;109(19):7517-7522.

9. Geoffroy CG, et al. Effects of PTEN and Nogo codeletion on corticospinal axon sprouting and regeneration in mice. J Neurosci. 2015;35(16):6413-6428.

10. Kadoya K, et al. Spinal cord reconstitution with homologous neural grafts enables robust corticospinal regeneration. Nat Med. 2016;22(5):479-487.

11. Liu K, et al. PTEN deletion enhances the regenerative ability of adult corticospinal neurons. Nat Neurosci. 2010;13(9):1075-1081.

12. Liu Y, et al. A Sensitized IGF1 Treatment Restores Corticospinal Axon-Dependent Functions. Neuron. 2017;95(4):817-833.e4.

13. O'Donovan KJ, et al. B-RAF kinase drives developmental axon growth and promotes axon regeneration in the injured mature CNS. JExp Med.
2014;211(5):801-814

14. Wu D, Klaw MC, Connors T, Kholodilov N, Burke RE, Tom VJ. Expressing constitutively active Rheb in adult neurons after a complete spinal cord injury enhances axonal regeneration beyond a chondroitinase-treated glial scar. J Neurosci. 2015;35(31):11068-11080.

15. Goodman CW, Brett AS. Gabapentin and pregabalin for pain - is increased prescribing a cause for concern? N Engl J Med. 2017;377(5):411-414.

16. Gee NS, Brown JP, Dissanayake VU, Offord J, Thurlow R, Woodruff GN. The novel anticonvulsant drug, gabapentin (Neurontin), binds to the alpha2delta subunit of a calcium channel. J Biol Chem. 1996;271(10):5768-5776.

17. Tedeschi A, et al. The calcium channel subunit $\alpha 2 \delta 2$ suppresses axon regeneration in the adult CNS. Neuron. 2016;92(2):419-434.

18. Feng $\mathrm{G}$, et al. Imaging neuronal subsets in transgenic mice expressing multiple spectral variants of GFP. Neuron. 2000;28(1):41-51.

19. Molyneaux BJ, et al. DeCoN: genome-wide analysis of in vivo transcriptional dynamics during 
pyramidal neuron fate selection in neocortex. Neuron. 2015;85(2):275-288.

20. Dolphin AC. Calcium channel auxiliary $\alpha 2 \delta$ and $\beta$ subunits: trafficking and one step beyond. Nat Rev Neurosci. 2012;13(8):542-555.

21. Li CY, et al. Calcium channel $\alpha 2 \delta 1$ subunit mediates spinal hyperexcitability in pain modulation. Pain. 2006;125(1-2):20-34.

22. Neely GG, et al. A genome-wide Drosophila screen for heat nociception identifies $\alpha 2 \delta 3$ as an evolutionarily conserved pain gene. Cell. 2010;143(4):628-638.

23. Xiong W, et al. Enhancing excitatory activity of somatosensory cortex alleviates neuropathic pain through regulating homeostatic plasticity. Sci Rep. 2017;7(1):12743.

24. Rosenzweig ES, et al. Restorative effects of human neural stem cell grafts on the primate spinal cord. Nat Med. 2018;24(4):484-490.

25. Davies A, et al. The calcium channel alpha2delta-2 subunit partitions with CaV2.1 into lipid rafts in cerebellum: implications for localization and function. J Neurosci. 2006;26(34):8748-8757.

26. Sofroniew MV. Dissecting spinal cord regeneration. Nature. 2018;557(7705):343-350.

27. Armbruster BN, Li X, Pausch MH, Herlitze S, Roth BL. Evolving the lock to fit the key to create a family of $G$ protein-coupled receptors potently activated by an inert ligand. Proc Natl Acad Sci US A. 2007;104(12):5163-5168.

28. Maier IC, Baumann K, Thallmair M, Weinmann O, Scholl J, Schwab ME. Constraint-induced movement therapy in the adult rat after unilateral corticospinal tract injury. J Neurosci. 2008;28(38):9386-9403.

29. Bregman BS, Kunkel-Bagden E, McAtee M, O'Neill A. Extension of the critical period for developmental plasticity of the corticospinal pathway. JComp Neurol. 1989;282(3):355-370.

30. Gribnau AA, de Kort EJ, Dederen PJ, Nieuwenhuys R. On the development of the pyramidal tract in the rat. II. An anterograde tracer study of the outgrowth of the corticospinal fibers. Anat Embryol. 1986;175(1):101-110.

31. Markram H, Toledo-Rodriguez M, Wang Y, Gupta A, Silberberg G, Wu C. Interneurons of the neocortical inhibitory system. Nat Rev Neurosci. 2004;5(10):793-807.

32. Turrigiano G. Homeostatic synaptic plasticity: local and global mechanisms for stabilizing neuronal function. Cold Spring Harb Perspect Biol. 2012;4(1):a005736.

33. Hoppa MB, Lana B, Margas W, Dolphin AC, Ryan TA. $\alpha 2 \delta$ Expression sets presynaptic calcium channel abundance and release probability. Nature. 2012;486(7401):122-125.

34. Gong HC, Hang J, Kohler W, Li L, Su TZ. Tissuespecific expression and gabapentin-binding properties of calcium channel $\alpha 2 \delta$ subunit subtypes. J Membr Biol. 2001;184(1):35-43.

35. Henley J, Poo MM. Guiding neuronal growth cones using $\mathrm{Ca}^{2+}$ signals. Trends Cell Biol. 2004;14(6):320-330.

36. Kater SB, Mattson MP, Cohan C, Connor J. Cal- cium regulation of the neuronal growth cone. Trends Neurosci. 1988;11(7):315-321.

37. Rehder V, Kater SB. Regulation of neuronal growth cone filopodia by intracellular calcium. J Neurosci. 1992;12(8):3175-3186.

38. Welnhofer EA, Zhao L, Cohan CS. Calcium influx alters actin bundle dynamics and retrograde flow in Helisoma growth cones. J Neurosci. 1999;19(18):7971-7982.

39. Bandtlow CE, Schmidt MF, Hassinger TD, Schwab ME, Kater SB. Role of intracellular calcium in NI-35-evoked collapse of neuronal growth cones. Science. 1993;259(5091):80-83.

40. Nicol X, Hong KP, Spitzer NC. Spatial and temporal second messenger codes for growth cone turning. Proc Natl Acad Sci U S A. 2011;108(33):13776-13781.

41. Snow DM, Atkinson PB, Hassinger TD, Letourneau PC, Kater SB. Chondroitin sulfate proteoglycan elevates cytoplasmic calcium in DRG neurons. Dev Biol. 1994;166(1):87-100.

42. Tedeschi A, et al. ADF/Cofilin-mediated actin turnover promotes axon regeneration in the adult CNS. Neuron. 2019;103(6):1073-1085.e6.

43. Bareyre FM, Kerschensteiner M, Raineteau O, Mettenleiter TC, Weinmann O, Schwab ME. The injured spinal cord spontaneously forms a new intraspinal circuit in adult rats. Nat Neurosci. 2004;7(3):269-277.

44. Courtine G, et al. Recovery of supraspinal control of stepping via indirect propriospinal relay connections after spinal cord injury. Nat Med. 2008;14(1):69-74.

45. Tuszynski MH, Steward O. Concepts and methods for the study of axonal regeneration in the CNS. Neuron. 2012;74(5):777-791.

46. Liu Y, et al. Touch and tactile neuropathic pain sensitivity are set by corticospinal projections. Nature. 2018;561(7724):547-550.

47. Ueno M, et al. Corticospinal circuits from the sensory and motor cortices differentially regulate skilled movements through distinct spinal interneurons. Cell Rep. 2018;23(5):1286-1300.e7.

48. Jiang YQ, Zaaimi B, Martin JH. Competition with primary sensory afferents drives remodeling of corticospinal axons in mature spinal motor circuits. J Neurosci. 2016;36(1):193-203.

49. Roth BL. DREADDs for neuroscientists. Neuron. 2016;89(4):683-694.

50. Moulin D, et al. Pharmacological management of chronic neuropathic pain: revised consensus statement from the Canadian Pain Society. Pain Res Manag. 2014;19(6):328-335.

51. Warner FM, et al. Early administration of gabapentinoids improves motor recovery after human spinal cord injury. Cell Rep. 2017;18(7):1614-1618.

52. Bauer CS, et al. The increased trafficking of the calcium channel subunit $\alpha 2 \delta$-1 to presynaptic terminals in neuropathic pain is inhibited by the $\alpha 2 \delta$ ligand pregabalin. J Neurosci. 2009;29(13):4076-4088.

53. Chen J, et al. The $\alpha 2 \delta-1-N M D A$ Receptor complex is critically involved in neuropathic pain devel- opment and gabapentin therapeutic actions. Cell Rep. 2018;22(9):2307-2321.

54. Luo ZD, et al. Injury type-specific calcium channel alpha 2 delta-1 subunit up-regulation in rat neuropathic pain models correlates with antiallodynic effects of gabapentin. JPharmacol Exp Ther. 2002;303(3):1199-1205.

55. Toth C. Pregabalin: latest safety evidence and clinical implications for the management of neuropathic pain. Ther Adv Drug Saf. 2014;5(1):38-56.

56. Lang BT, et al. Modulation of the proteoglycan receptor PTP $\sigma$ promotes recovery after spinal cord injury. Nature. 2015;518(7539):404-408.

57. Warren PM, Steiger SC, Dick TE, MacFarlane PM, Alilain WJ, Silver J. Rapid and robust restoration of breathing long after spinal cord injury. Nat Commun. 2018;9(1):4843.

58. Devillard X, Rimaud D, Roche F, Calmels P. Effects of training programs for spinal cord injury. Ann Readapt Med Phys. 2007;50(6):490-498, 480.

59. Torres-Espín A, Beaudry E, Fenrich K, Fouad K. Rehabilitative training in animal models of spinal cord injury. J Neurotrauma. 2018;35(16):1970-1985.

60. Wahl AS, et al. Neuronal repair. Asynchronous therapy restores motor control by rewiring of the rat corticospinal tract after stroke. Science. 2014;344(6189):1250-1255.

61. Hendrich J, Bauer CS, Dolphin AC. Chronic pregabalin inhibits synaptic transmission between rat dorsal root ganglion and dorsal horn neurons in culture. Channels (Austin). 2012;6(2):124-132.

62. Patel MK, Gonzalez MI, Bramwell S, Pinnock RD, Lee K. Gabapentin inhibits excitatory synaptic transmission in the hyperalgesic spinal cord. $\mathrm{BrJ}$ Pharmacol. 2000;130(8):1731-1734.

63. Tedeschi A, et al. The tumor suppressor p53 transcriptionally regulates cGKI expression during neuronal maturation and is required for cGMP-dependent growth cone collapse. J Neurosci. 2009;29(48):15155-15160.

64. Lewicki MS. A review of methods for spike sorting: the detection and classification of neural action potentials. Network. 1998;9(4):R53-R78.

65. Pouzat C, Mazor O, Laurent G. Using noise signature to optimize spike-sorting and to assess neuronal classification quality. JNeurosci Methods. 2002;122(1):43-57.

66. Rey HG, Pedreira C, Quian Quiroga R. Past, present and future of spike sorting techniques. Brain Res Bull. 2015;119(pt B):106-117.

67. Susaki EA, Tainaka K, Perrin D, Yukinaga H, Kuno A, Ueda HR. Advanced CUBIC protocols for whole-brain and whole-body clearing and imaging. Nat Protoc. 2015;10(11):1709-1727.

68. Longair MH, Baker DA, Armstrong JD. Simple Neurite Tracer: open source software for reconstruction, visualization and analysis of neuronal processes. Bioinformatics. 2011;27(17):2453-2454.

69. Schmid B, Schindelin J, Cardona A, Longair M, Heisenberg M. A high-level 3D visualization API for Java and ImageJ. BMC Bioinformatics. 2010;11:274.

70. Schindelin J, et al. Fiji: an open-source platform for biological-image analysis. Nat Methods. 2012;9(7):676-682. 